Article

\title{
Concave-Convex Problems for the Robin $p$-Laplacian Plus an Indefinite Potential
}

\author{
Nikolaos S. Papageorgiou ${ }^{1}$ and Andrea Scapellato ${ }^{2, *}$ ii \\ 1 Department of Mathematics, National Technical University, Zografou Campus, 15780 Athens, Greece; \\ npapg@math.ntua.gr \\ 2 Dipartimento di Matematica e Informatica, Università degli Studi di Catania, Viale Andrea Doria 6, \\ 95125 Catania, Italy \\ * Correspondence: scapellato@dmi.unict.it
}

Received: 15 February 2020; Accepted: 11 March 2020; Published: 14 March 2020

check for updates

\begin{abstract}
We consider nonlinear Robin problems driven by the $p$-Laplacian plus an indefinite potential. In the reaction, we have the competing effects of a parametric concave (that is, $(p-$ 1)-sublinear) term and of a convex (that is, $(p-1)$-superlinear) term which need not satisfy the Ambrosetti-Rabinowitz condition. We prove a "bifurcation-type" theorem describing in a precise way the dependence the dependence of the set of positive solutions on the parameter $\lambda>0$. In addition, we show the existence of a smallest positive solution $u_{\lambda}^{*}$ and determine the monotonicity and continuity properties of the map $\lambda \mapsto u_{\lambda}^{*}$.
\end{abstract}

Keywords: concave-convex nonlinearities; $p$-Laplacian; indefinite potential; antimaximum principle; nonlinear regularity; positive solutions

\section{Introduction}

Let $\Omega \subseteq \mathbb{R}^{N}$ be a bounded domain with a $C^{2}$-boundary $\partial \Omega$. In this paper, we study the following nonlinear parametric Robin problem

$$
\begin{cases}-\Delta_{p} u(z)+\xi(z) u(z)^{p-1}=\lambda g(z, u(z))+f(z, u(z)) & \text { in } \Omega \\ \frac{\partial u}{\partial n_{p}}+\beta(z) u^{p-1}=0 & \text { on } \partial \Omega \\ u>0 & \end{cases}
$$

with $1<p<\infty, \lambda>0$.

In this problem, $\Delta_{p}$ denotes the $p$-Laplace differential operator defined by

$$
\Delta_{p} u=\operatorname{div}\left(|D u|^{p-2} D u\right) \quad \text { for all } u \in W^{1, p}(\Omega), 1<p<\infty .
$$

The potential function $\xi \in L^{\infty}(\Omega)$ and in general it is sign changing. Thus, the left-hand side of (1) (the differential operator plus the potential term) is not coercive. On the right-hand side of the problem (the reaction), we have the competing effects of two nonlinearities. One is the parametric term $\lambda g(z, x)$ with $g(\cdot, \cdot)$ being a Carathéodory function (that is, for all $x \in \mathbb{R}, z \mapsto g(z, x)$ is measurable and for a.a. $z \in \Omega, x \mapsto g(z, x)$ is continuous). The function $g(z, \cdot)$ is strictly $(p-1)$-sublinear near $+\infty$ and concave near the origin (that is, $(p-1)$-superlinear near zero). The second nonlinearity $f(z, x)$ is also a Carathéodory function and $f(z, \cdot)$ is $(p-1)$-superlinear near $+\infty$. The superlinearity of $f(z, \cdot)$ is not expressed using the well-known Ambrosetti-Rabinowitz condition (the AR-condition for short). Instead, we employ a less restrictive condition which incorporates in our framework a larger class 
of $(p-1)$-superlinear functions. In the boundary condition, $\frac{\partial u}{\partial n_{p}}$ denotes the conormal derivative of $u$ corresponding to the $p$-Laplacian and it is interpreted via the nonlinear Green's identity (see Gasiński-Papageorgiou [1], p. 211).

In particular,

$$
\frac{\partial u}{\partial n_{p}}=|D u|^{p-2}(D u, n)_{\mathbb{R}^{N}}, \quad \text { for all } u \in C^{1}(\bar{\Omega}),
$$

with $n(\cdot)$ being the outward unit normal on $\partial \Omega$. The boundary coefficient $\beta(\cdot)$ is nonnegative and can be identically zero (Neumann problem).

From the above description, we see that problem (1) is a generalized concave-convex nonlinear Robin problem. We look for positive solutions of (1) and our goal is to describe in detail the changes in the set of positive solutions of (1) as the parameter $\lambda>0$ varies in the open positive semiaxis $\mathbb{R}_{+}=(0,+\infty)$.

Thus, we prove a "bifurcation-type" theorem, according to which there exists a critical parameter $\lambda^{*}>0$ such that

- for all $\lambda \in\left(0, \lambda^{*}\right)$ problem (1) has at least two positive solutions;

- $\quad$ for $\lambda=\lambda^{*}$ problem (1) has at least one positive solution;

- for all $\lambda>\lambda^{*}$ there are no positive solutions for problem (1).

In addition, we show that, for every admissible parameter $\lambda \in \mathscr{L}=\left(0, \lambda^{*}\right]$, problem (1) has a smallest positive solution $u_{\lambda}^{*}$, and we also examine the monotonicity and continuity properties of the $\operatorname{map} \mathscr{L} \ni \lambda \mapsto u_{\lambda}^{*}$.

Our work here extends to nonlinear problems driven by the $p$-Laplacian the recent semilinear work of Papageorgiou-Rădulescu-Repovš [2]. An inspection of their method of proof reveals that it is heavily dependent on the fact that the Sobolev space $H^{1}(\Omega)$ can be written as the orthogonal direct sum of the eigenspaces of $-\Delta+\xi(z) I$ with Robin boundary condition. No such decomposition is available for $W^{1, p}(\Omega)$ in the case of the $p$-Laplacian. Moreover, in the semilinear case strong comparison principles are an easy consequence of the Hopf boundary point theorem. In contrast, in the nonlinear case, it is much more difficult to come up with strong comparison principles and stronger conditions are needed. We point out that our conditions on the two nonlinearities $g(z, x)$ and $f(z, x)$, are in general less restrictive than those in [2]. Finally, we mention the recent work of Marano-Marino-Papageorgiou [3] on Dirichlet problems with no potential term and a more restrictive reaction.

The study of parametric concave-convex problem (as they are usually called in the literature problems exhibiting the competing effects on sublinear and superlinear nonlinearities), started with the seminal paper of Ambrosetti-Brezis-Cerami [4], which deals with semilinear Dirichlet problems driven by the Laplacian. Their work was extended to equations driven by the Dirichlet $p$-Laplacian by García Azorero-Manfredi-Peral Alonso [5] and Guo-Zhang [6]. In these works, the reaction has the particular form

$$
x \mapsto \lambda x^{q-1}+x^{r-1} \text { for all } x>0 \text {, with } 1<q<p<r<p^{*}, \lambda>0 .
$$

Here, $p^{*}$ denotes the critical Sobolev exponent corresponding to $p$, that is,

$$
p^{*}=\left\{\begin{array}{ll}
\frac{N p}{N-p} & \text { if } p<N \\
+\infty & \text { if } N \leq p
\end{array} .\right.
$$

More general reactions can be found in the works of $\mathrm{Hu}$-Papageorgiou [7] and Marano-Papageorgiou [8]. In all these works, $\xi \equiv 0$ and the differential operator is coercive. Finally, we mention the recent work of Papageorgiou-Scapellato [9] dealing with a similar parametric Robin problem in which the reaction term is $(p-1)$-linear and exhibits an asymmetric behavior as $x \rightarrow \pm \infty$. The authors produce nodal solutions (see also [10]). 
It is worth pointing out that many other researchers studied differential problems close to those considered in this paper. DiBenedetto-Gianazza-Vespri [11] studied the local boundedness and the local Hölder continuity of local weak solutions to anisotropic $p$-Laplacian type equations. Drábek-Hernández [12] studied quasilinear eigenvalue problems with singular weights driven by the $p$-Laplacian. Drábek-Ho-Sarkar considered an eigenvalue problem involving the weighted $p$-Laplacian in radially symmetric domains [13] and the Fredholm alternative for the $p$-Laplacian in exterior domains [14]. Drábek-Pohozaev [15], using the fibrering method, proved the existence of multiple positive solutions to quasilinear problems of second order driven by the $p$-Laplacian and also proved nonexistence results. Jebelean-Mawhin-Şerban [16] considered a system of difference equations with periodic nonlinearities and applying a modification argument to a suitable problem with a left-hand member of $p$-Laplacian type and using Morse theory, obtained multiple periodic solutions. Finally, we mention the work of Manásevich-Mawhin [17] that deals with the spectrum of $p$-Laplacian-type systems with certain boundary conditions.

\section{Mathematical Background-Hypotheses}

In the study of problem (1), there are three main spaces that we will use: the Sobolev space $W^{1, p}(\Omega)$, the Banach space $C^{1}(\bar{\Omega})$, and the boundary Lebesgue spaces $L^{q}(\partial \Omega)(1 \leq q \leq \infty)$.

The symbol $\|\cdot\|$ denotes the norm of $W^{1, p}(\Omega)$, defined by

$$
\|u\|=\left[\|u\|_{p}^{p}+\|D u\|_{p}^{p}\right]^{\frac{1}{p}} \quad \text { for all } u \in W^{1, p}(\Omega) .
$$

The Banach space $C^{1}(\bar{\Omega})$ is ordered by the positive (order) cone

$$
C_{+}=\left\{u \in C^{1}(\bar{\Omega}): u(z) \geq 0 \text { for all } z \in \bar{\Omega}\right\} .
$$

This cone has a nonempty interior given by

$$
\operatorname{int} C_{+}=\left\{u \in C_{+}: u(z)>0 \text { for all } z \in \bar{\Omega}\right\} .
$$

We will also use another open cone in $C^{1}(\bar{\Omega})$, namely

$$
\widehat{K}_{+}=\left\{u \in C^{1}(\bar{\Omega}): u(z)>0 \text { for all } z \in \bar{\Omega},\left.\frac{\partial u}{\partial n}\right|_{\partial \Omega \cap u^{-1}(0)}<0\right\} .
$$

On $\partial \Omega$, we consider the $(N-1)$-dimensional Hausdorff (surface) measure $\sigma(\cdot)$. Having this measure on $\partial \Omega$, we can define in the usual way the boundary Lebesgue spaces $L^{q}(\partial \Omega)(1 \leq q \leq \infty)$. It is known that there exists a unique continuous linear map $\gamma_{0}: W^{1, p}(\Omega) \rightarrow L^{p}(\partial \Omega)$, called the trace map, such that

$$
\gamma_{0}(u)=\left.u\right|_{\partial \Omega} \text { for all } u \in W^{1, p}(\Omega) \cap C(\bar{\Omega}) .
$$

We know that

(a) $\quad \gamma_{0}\left(W^{1, p}(\Omega)\right)=W^{\frac{1}{p^{\prime}}, p}(\partial \Omega)\left(\frac{1}{p}+\frac{1}{p^{\prime}}=1\right)$ and $\operatorname{ker} \gamma_{0}=W_{0}^{1, p}(\Omega)$.

(b) $\quad \gamma_{0}$ is compact into $L^{q}(\partial \Omega)$ for all $q \in\left[1, \frac{p(N-1)}{N-p}\right)$ if $p<N$ and into $L^{q}(\partial \Omega)$ for all $q \in[1, \infty)$ if $N \leq p$.

In the sequel for notational economy, we drop the use of $\gamma_{0}$. All restrictions of Sobolev functions on $\partial \Omega$, are understood in the sense of traces.

For $v, u \in W^{1, p}(\Omega)$ with $v \leq u$, we define

$$
[v, u]=\left\{y \in W^{1, p}(\Omega): v(z) \leq y(z) \leq u(z) \text { for a.a. } z \in \Omega\right\} .
$$


In addition, by $\operatorname{int}_{C^{1}(\bar{\Omega})}[v, u]$, we denote the interior in the $C^{1}(\bar{\Omega})$ - norm topology of $[v, u] \cap C^{1}(\bar{\Omega})$. Moreover, for any $\widehat{u} \in W^{1, p}(\Omega)$, we define

$$
[\widehat{u})=\left\{y \in W^{1, p}(\Omega): \widehat{u}(z) \leq y(z) \text { for a.a. } z \in \Omega\right\} .
$$

For every $x \in \mathbb{R}$, we set $x^{ \pm}=\max \{ \pm x, 0\}$ and then for any $u \in W^{1, p}(\Omega)$, we define $u^{ \pm}(\cdot)=u(\cdot)^{ \pm}$. We know that

$$
u^{ \pm} \in W^{1, p}(\Omega), \quad u=u^{+}-u^{-}, \quad|u|=u^{+}+u^{-} .
$$

Given $X$ a Banach space, by $X^{*}$, we denote its topological dual and with the symbol $\langle\cdot, \cdot\rangle$ we denote the duality brackets for the pair $\left(X^{*}, X\right)$. If $\varphi \in C^{1}(X, \mathbb{R})$, then we say that $\varphi(\cdot)$ satisfies the Cerami condition (the $C$-condition for short), if the following property holds:

Every sequence $\left\{u_{n}\right\}_{n \geq 1} \subseteq X$ such that

$$
\begin{gathered}
\left\{\varphi\left(u_{n}\right)\right\}_{n \geq 1} \subseteq \mathbb{R} \text { is bounded, } \\
\left(1+\left\|u_{n}\right\|\right) \varphi^{\prime}\left(u_{n}\right) \rightarrow 0 \text { in } X^{*} \text { as } n \rightarrow \infty,
\end{gathered}
$$

admits a strongly convergent subsequence.

By $K_{\varphi}$, we denote the critical set of $\varphi$, that is,

$$
K_{\varphi}=\left\{u \in X: \varphi^{\prime}(u)=0\right\} .
$$

In addition, a map $A: X \rightarrow X^{*}$ is said to be an $(S)_{+}$-map if it has the following property (see [18], p. 203):

$$
u_{n} \stackrel{w}{\rightarrow} u \text { in } X \text { and } \limsup _{n \rightarrow \infty}\left\langle A\left(u_{n}\right), u_{n}-u\right\rangle \leq 0 \quad \Rightarrow \quad u_{n} \rightarrow u \text { in } X
$$

Let $A: W^{1, p}(\Omega) \rightarrow W^{1, p}(\Omega)^{*}$ be defined by

$$
\langle A(u), h\rangle=\int_{\Omega}|D u|^{p-2}(D u, D h)_{\mathbb{R}^{N}} \mathrm{~d} z \quad \text { for all } u, h \in W^{1, p}(\Omega) .
$$

Proposition 1. The nonlinear map $A: W^{1, p}(\Omega) \rightarrow W^{1, p}(\Omega)^{*}$ defined above is bounded (that is, it maps bounded sets to bounded sets), continuous, monotone (hence maximal monotone too) and of type $(S)_{+}$.

Now, we introduce our hypotheses on the data of problem (1).

$\mathrm{H}(\xi): \quad \xi \in L^{\infty}(\Omega)$

Remark 1. We see that $\xi(\cdot)$ is not nonnegative. This makes the left-hand side of (1) noncoercive, a feature of the problem that makes its analysis more difficult.

$\mathrm{H}(\beta): \quad \beta \in C^{0, \alpha}(\partial \Omega)$ with $\alpha \in(0,1), \beta(z) \geq 0$ for all $z \in \partial \Omega$.

Remark 2. When $\beta \equiv 0$, we recover the Neumann problem. The regularity requirements on $\beta(\cdot)$ will be used to have global regularity results for the produced solutions.

$\mathrm{H}(g): \quad g: \Omega \times \mathbb{R} \rightarrow \mathbb{R}$ is a Carathéodory function such that $g(z, 0)=0$ for a.a. $z \in \Omega$ and

(i) for every $\rho>0$ there exists $a_{\rho} \in L^{\infty}(\Omega)$ such that

$$
0 \leq g(z, x) \leq a_{\rho}(z) \text { for a.a. } z \in \Omega, 0 \leq x \leq \rho ;
$$


(ii) $\lim _{x \rightarrow+\infty} \frac{g(z, x)}{x^{p-1}}=0$ uniformly for a.a. $z \in \Omega$;

(iii) there exist $q \in(1, p)$ and $c_{0}, c_{1}, \widehat{c}, \delta_{0}>0$ such that

$$
\begin{gathered}
c_{0} x^{q-1} \leq g(z, x) \quad \text { for a.a. } z \in \Omega, \text { all } 0 \leq x \leq \delta_{0}, \\
\limsup _{x \rightarrow 0^{+}} \frac{g(z, x)}{x^{q-1}} \leq c_{1} \quad \text { uniformly for a.a. } z \in \Omega, \\
\limsup _{x \rightarrow+\infty} \frac{p G(z, x)-g(z, x) x}{x^{q}} \leq \widehat{c} \quad \text { uniformly for a.a. } z \in \Omega,
\end{gathered}
$$

with $G(z, x)=\int_{0}^{x} g(z, s) \mathrm{d} s$.

(iv) for every $s>0$, we have $g(z, x) \geq m_{s}>0$ for a.a. $z \in \Omega$, all $x \geq s$.

Remark 3. Since we look for positive solutions and the above hypotheses concern the positive semiaxis $\mathbb{R}_{+}=[0,+\infty)$, without any loss of generality, we may assume that

$$
g(z, x)=0 \text { for a.a. } z \in \Omega \text {, all } x \leq 0 .
$$

Hypothesis $H(g)$ (ii) implies that, for a.a. $z \in \Omega$, the nonlinearity $x \mapsto g(z, x)$ is strictly $(p-1)$-sublinear near $+\infty$, while hypothesis $H(g)$ (iii) implies that near $0^{+}$there is a local $(p-1)$-superlinear term. Therefore, $g(z, \cdot)$ is a generalized "concave" nonlinearity.

$\mathrm{H}(f): \quad f: \Omega \times \mathbb{R} \rightarrow \mathbb{R}$ is a Carathéodory function such that $f(z, 0)=0$ for a.a. $z \in \Omega$ and

(i) $\quad 0 \leq f(z, x) \leq a(z)\left[1+x^{r-1}\right]$ for a.a. $z \in \Omega$, all $x \geq 0$, with $a \in L^{\infty}(\Omega), p<r<p^{*}$;

(ii) if $F(z, x)=\int_{0}^{x} f(z, s) \mathrm{d} s$, then $\lim _{x \rightarrow+\infty} \frac{F(z, x)}{x^{p}}=+\infty$ uniformly for a.a. $z \in \Omega$;

(iii) there exists $\tau \in\left(\max \left\{1, \frac{N}{p}\right\}(r-p), p^{*}\right), \tau>q$ (see hypothesis $\mathrm{H}(g)$ (iii)) such that

$$
0 \leq \widehat{c}_{0} \leq \liminf _{x \rightarrow+\infty} \frac{f(z, x) x-p F(z, x)}{x^{\tau}} \quad \text { uniformly for a.a. } z \in \Omega ;
$$

(iv) $\lim _{x \rightarrow 0^{+}} \frac{f(z, x)}{x^{q-1}}=0$ uniformly for a.a. $z \in \Omega$;

(v) there exist $c_{2}, c_{3}, c_{4}>0$ and $\vartheta \in(q, p)$ such that

$$
f(z, x) \leq c_{2} x^{r-1}+c_{3} x^{\vartheta-1}-c_{4} x^{p-1} \quad \text { for a.a. } z \in \Omega \text {, all } x \geq 0 .
$$

Remark 4. As we did for the nonlinearity $g(z, \cdot)$, we may assume that

$$
f(z, x)=0 \text { for a.a. } z \in \Omega \text {, all } x \leq 0 .
$$

From hypotheses $H(f)$ (ii),(iii), we infer that

$$
\lim _{x \rightarrow+\infty} \frac{f(z, x)}{x^{p-1}}=+\infty \quad \text { uniformly for a.a. } z \in \Omega .
$$


Thus, the perturbation term $f(z, \cdot)$ is $(p-1)$-superlinear. In the literature, equations having a $(p-1)$-superlinear reaction are usually treated using the AR-condition, which says that there exist $\eta>p$ and $M>0$ such that

$$
\begin{aligned}
& 0<\eta F(z, x) \leq f(z, x) x \quad \text { for a.a. } z \in \Omega, \text { all } x \geq M, \\
& 0<\underset{\Omega}{\operatorname{essinf}} F(\cdot, M)
\end{aligned}
$$

(see Ambrosetti-Rabinowitz [19]). Actually, this is a unilateral version of the AR-condition since we have assumed (2) and (3). Integrating (4a) and using (4b), we obtain a weaker condition, namely that

$$
\begin{aligned}
& c_{5} x^{\eta} \leq F(z, x) \quad \text { for a.a. } z \in \Omega \text {, all } x \geq M \text {, some } c_{5}>0, \\
\Rightarrow & c_{5} x^{\eta-1} \leq f(z, x) \quad \text { for a.a. } z \in \Omega \text {, all } x \geq M .
\end{aligned}
$$

Therefore, the AR-condition implies that $f(z, \cdot)$ has at least $(\eta-1)$-polynomial growth. The AR-condition although convenient in the verification of the C-condition for the energy (Euler) functional of the problem, it is rather restrictive and excludes from consideration superlinear nonlinearities with slower growth near $+\infty$ (see the Examples below). Here, instead, we use the less restrictive condition $H(f)$ (iii). Note that, if $f(z, \cdot)$ satisfies the AR-condition, then we may assume that $\eta>\max \left\{1,(r-p) \frac{N}{p}\right\}$ and we have

$$
\frac{f(z, x) x-p F(z, x)}{x^{\eta}}=\frac{f(z, x) x-\eta F(z, x)}{x^{\eta}}+(\eta-p) \frac{f(z, x)}{x^{\eta}} \geq(\vartheta-p) c_{5}
$$

and so hypothesis $H(f)$ (iii) is satisfied. Hypothesis $H(f)$ (iv) implies that $f(z, \cdot)$ is $(q-1)$-sublinear near $0^{+}$. Thus, the behavior of $f(z, \cdot)$ both near $+\infty$ and near $0^{+}$is complementary to that of $g(z, \cdot)$.

$\mathrm{H}_{0}$ : $\quad$ For every $\rho>0$ and every $B \subseteq(0,+\infty)$ bounded, there exists $\widehat{\xi}_{\rho}^{B}>0$ such that for a.a. $z \in \Omega$, all $\lambda \in B$, the function

$$
x \mapsto \lambda g(z, x)+f(z, x)+\widehat{\xi}_{\rho}^{B} x^{p-1}
$$

is nondecreasing on $[0, \rho]$.

Remark 5. If for a.a. $z \in \Omega$ the functions $g(z, \cdot)$ and $f(z, \cdot)$ are differentiable and for every $\rho>0$ and $B \subseteq(0,+\infty)$ bounded, we have

$$
\left[\lambda g_{x}^{\prime}(z, x)+f_{x}^{\prime}(z, x)\right] x \geq-(p-1) \widehat{\xi}_{\rho}^{B} x^{p-1} \quad \text { for a.a. } z \in \Omega \text {, all } \lambda \in B, \text { with } \widehat{\xi}_{\rho}^{B}>0
$$

then hypothesis $\mathrm{H}_{0}$ is satisfied.

Example 1. The following pairs of function satisfy hypotheses $H(g), H(f), \mathrm{H}_{0}$. For the sake of simplicity, we drop the z-dependence:

$$
\begin{aligned}
& g_{1}(x)=x^{q-1}, 1<q<p, \quad f_{1}(x)=x^{r-1}, p<r<p^{*}, \text { for all } x \geq 0, \\
& g_{2}(x)=\left\{\begin{array}{ll}
x^{\eta-1} & \text { if } 0 \leq x \leq 1 \\
x^{q-1} & \text { if } 1<x
\end{array}, \eta, q<p, \quad f_{2}(x)=x^{p-1} \ln (1+x), \text { for all } x \geq 0 .\right.
\end{aligned}
$$

Note that the pair $\left(g_{1}, f_{1}\right)$ is the classical "concave-convex" pair with $f_{1}$ satisfying the AR-condition. On the other hand, $f_{2}$ does not satisfy the AR-condition. 


\section{Positive Solutions}

We introduce the following sets

$$
\begin{aligned}
& \mathscr{L}=\{\lambda>0: \text { problem (1) admits a positive solution }\}, \quad \text { (set of admissible parameters), } \\
& \mathscr{S}_{\lambda}=\text { set of positive solutions of }(1) .
\end{aligned}
$$

In addition, we define

$$
\lambda^{*}=\sup \mathscr{L}
$$

Suppose that $u \in \mathscr{S}_{\lambda}$. From Proposition 2.10 of Papageorgiou-Rădulescu [20], we have $u \in$ $L^{\infty}(\Omega)$. Then, Theorem 2 of Lieberman [21] implies that $u \in C_{+} \backslash\{0\}$. Let $\rho=\|u\|_{\infty}, B=\{\lambda\}$ and $\widehat{\xi}_{\rho}^{B}>0$ be as postulated by hypothesis $\mathrm{H}_{0}$. We have

$$
\begin{aligned}
& -\Delta_{p} u(z)+\left[\xi(z)+\widehat{\xi}_{\rho}^{B}\right] u(z)^{p-1} \geq 0 \quad \text { for a.a. } z \in \Omega, \\
\Rightarrow & \Delta_{p} u(z) \leq\left[\|\xi\|_{\infty}+\widehat{\zeta}_{\rho}^{B}\right] u(z)^{p-1} \quad \text { for a.a. } z \in \Omega, \\
\Rightarrow & u \in \operatorname{int} C_{+},
\end{aligned}
$$

The fact that $u \in \operatorname{int} C_{+}$follows from Pucci-Serrin [22] (pp. 111, 120).

Thus, we have proved that

$$
\mathscr{S}_{\lambda} \subseteq \operatorname{int} C_{+} \text {for all } \lambda>0
$$

\subsection{Nonemptiness of $\mathscr{L}$}

In this subsection, we show that $\mathscr{L} \neq \varnothing$; namely, we show that there exist admissible parameters. In what follows by $\gamma_{p}: W^{1, p}(\Omega) \rightarrow \mathbb{R}$, we denote the $C^{1}$-functional defined by

$$
\gamma_{p}(u)=\|D u\|_{p}^{p}+\int_{\Omega} \xi(z)|u|^{p} \mathrm{~d} z+\int_{\partial \Omega} \beta(z)|u|^{p} \mathrm{~d} \sigma \quad \text { for all } u \in W^{1, p}(\Omega)
$$

Proposition 2. If hypotheses $\mathrm{H}(\xi), \mathrm{H}(\beta), \mathrm{H}(g), \mathrm{H}(f), \mathrm{H}_{0}$ hold, then $\mathscr{L} \neq \varnothing$.

Proof. Let $\eta>\|\xi\|_{\infty}$ and consider the $C^{1}$-functional $\hat{\varphi}_{\lambda}: W^{1, p}(\Omega) \rightarrow \mathbb{R}$ defined by

$$
\widehat{\varphi}_{\lambda}(u)=\frac{1}{p} \gamma_{p}(u)+\frac{\eta}{p}\left\|u^{-}\right\|_{p}^{p}-\lambda \int_{\Omega} G\left(z, u^{+}\right) \mathrm{d} z-\int_{\Omega} F\left(z, u^{+}\right) \mathrm{d} z \quad \text { for all } u \in W^{1, p}(\Omega) .
$$

From hypothesis $\mathrm{H}(f)(\mathrm{v})$, we have

$$
F(z, x) \leq \frac{c_{2}}{r} x^{r}+\frac{c_{3}}{\vartheta} x^{\vartheta}-\frac{c_{4}}{p} x^{p} \quad \text { for a.a. } z \in \Omega, \text { all } x \geq 0
$$

Note that, by appropriately modifying $c_{2}>0$ and $c_{3}>0$ if necessary, we may assume that

$$
c_{4}>\|\xi\|_{\infty}
$$

(recall that $\vartheta<p<r$ ). Since $q<\vartheta<p<r$, given $\epsilon>0$, we can find $c_{6}=c_{6}(\epsilon)>0$ such that

$$
\frac{c_{3}}{\vartheta} x^{\vartheta} \leq \frac{\epsilon}{q} x^{q}+\frac{c_{6}}{r} x^{r} \quad \text { for all } x \geq 0
$$

Moreover, hypotheses $\mathrm{H}(g)$ imply that given $\epsilon>0$, we can find $c_{7}=c_{7}(\epsilon)>0$ such that

$$
G(z, x) \leq \frac{c_{7}}{q} x^{q}+\frac{\epsilon}{p} x^{p} \quad \text { for a.a. } z \in \Omega, \text { all } x \geq 0 .
$$


Then, for every $u \in W^{1, p}(\Omega)$, we have

$$
\begin{aligned}
\widehat{\varphi}_{\lambda}(u) \geq & \frac{1}{p}\left[\gamma_{p}\left(u^{-}\right)+\eta\left\|u^{-}\right\|_{p}^{p}\right]+\frac{1}{p}\left[\gamma_{p}\left(u^{+}\right)+\left(c_{4}-\lambda \epsilon\right)\left\|u^{+}\right\|_{p}^{p}\right] \\
& -\frac{c_{6}}{r}\|u\|^{r}-c_{8}(\epsilon+\lambda)\|u\|^{q} \quad \text { for some } c_{8}>0 \text { (independent of } \epsilon>0 \text { ). }
\end{aligned}
$$

Thus, given $\tilde{\lambda}>0$, if $\lambda \in(0, \tilde{\lambda})$, then choosing $\epsilon \in\left(0, \frac{c_{4}}{\tilde{\lambda}}\right)$, we have

$$
\begin{aligned}
\widehat{\varphi}_{\lambda}(u) \geq & c_{9}\|u\|^{p}-c_{10}\|u\|^{r}-c_{8}(\epsilon+\lambda)\|u\|^{q} \\
& \text { for some } c_{9}>0\left(\text { recall that } \eta>\|\xi\|_{\infty}\right) \text { and with } c_{10}=\frac{c_{6}}{r}>0 \\
= & {\left[c_{9}-c_{10}\|u\|^{r-p}+c_{8}(\epsilon+\lambda)\|u\|^{q-p}\right]\|u\|^{p} } \\
& \quad \text { for all } u \in W^{1, p}(\Omega), \text { all } \lambda \in(0, \widetilde{\lambda}) .
\end{aligned}
$$

We consider the function

$$
\tau_{\lambda}(t)=c_{10} t^{r-p}+c_{8}(\epsilon+\lambda) t^{q-p} \quad \text { for all } t>0 .
$$

It is clear that $\tau_{\lambda} \in C^{1}(0, \infty)$ and, since $1<q<p<r$, we have

$$
\tau_{\lambda}(t) \rightarrow+\infty \quad \text { as } t \rightarrow 0^{+} \text {and as } t \rightarrow+\infty .
$$

Therefore, we can find $t_{0} \in(0, \infty)$ such that

$$
\begin{aligned}
& \tau_{\lambda}\left(t_{0}\right)=\min \left[\tau_{\lambda}(t): t>0\right], \\
\Rightarrow & \tau_{\lambda}^{\prime}\left(t_{0}\right)=0, \\
\Rightarrow & c_{8}(\epsilon+\lambda)(p-q) t_{0}^{q-p-1}=c_{10}(r-p) t_{0}^{r-p-1}, \\
\Rightarrow & t_{0}=t_{0}(\lambda)=\left[\frac{c_{8}(\epsilon+\lambda)(p-q)}{c_{10}(r-p)}\right]^{\frac{1}{r-q}} .
\end{aligned}
$$

Then, we have

$$
\tau_{\lambda}\left(t_{0}\right)=c_{10}\left[\frac{c_{8}(\epsilon+\lambda)(p-q)}{c_{10}(r-p)}\right]^{\frac{r-p}{r-q}}+c_{8}(\epsilon+\lambda)^{\frac{r-p}{r-q}}\left[\frac{c_{10}(r-p)}{c_{8}(p-q)}\right]^{\frac{p-q}{r-q}} .
$$

It follows that

$$
\tau_{\lambda}\left(t_{0}\right) \rightarrow c_{11} \epsilon^{\frac{r-p}{r-q}} \text { as } \lambda \rightarrow 0^{+} \text {with } c_{11}>0 \text { (independent of } \epsilon>0 \text { ). }
$$

Given $\delta>0$, we can find $\lambda_{0} \in(0, \widetilde{\lambda}]$ such that

$$
\tau_{\lambda}\left(t_{0}\right) \leq c_{11} \epsilon^{\frac{r-p}{r-q}}+\delta \quad \text { for all } \lambda \in\left(0, \lambda_{0}\right]
$$

Since $\epsilon \in\left(0, \frac{c_{4}}{\tilde{\lambda}}\right)$ and $\delta>0$ are arbitrary, we can choose both small so that

$$
\begin{aligned}
& \tau_{\lambda}\left(t_{0}\right) \leq c_{11} \epsilon^{\frac{r-p}{r-q}}+\delta<c_{9} \quad \text { for all } \lambda \in\left(0, \lambda_{0}\right], \\
\Rightarrow \quad & \widehat{\varphi}_{\lambda}(u)>0=\widehat{\varphi}_{\lambda}(0) \quad \text { for all }\|u\|=t_{0}(\lambda)(\text { see }(10)) .
\end{aligned}
$$

Hypothesis $\mathrm{H}(f)$ (ii) implies that, if $u \in \operatorname{int} C_{+}$, then, for every $\lambda>0$,

$$
\widehat{\varphi}_{\lambda}(t u) \rightarrow-\infty \quad \text { as } t \rightarrow+\infty .
$$


Claim: For every $\lambda>0$, the functional $\widehat{\varphi}_{\lambda}(\cdot)$ satisfies the $C$-condition.

We consider a sequence $\left\{u_{n}\right\}_{n \geq 1} \subseteq W^{1, p}(\Omega)$ such that

$$
\begin{aligned}
& \left|\widehat{\varphi}_{\lambda}\left(u_{n}\right)\right| \leq M_{1} \text { for some } M_{1}>0, \text { all } n \in \mathbb{N}, \\
& \left(1+\left\|u_{n}\right\|\right) \widehat{\varphi}_{\lambda}^{\prime}\left(u_{n}\right) \rightarrow 0 \quad \text { in } W^{1, p}(\Omega)^{*} \text { as } n \rightarrow \infty .
\end{aligned}
$$

From (14), we have

$$
\begin{gathered}
\left.\left|\left\langle A\left(u_{n}\right), h\right\rangle+\int_{\Omega} \xi(z)\right| u_{n}\right|^{p-2} u_{n} h \mathrm{~d} z+\int_{\partial \Omega} \beta(z)\left|u_{n}\right|^{p-2} u_{n} h \mathrm{~d} \sigma-\eta \int_{\Omega}\left(u_{n}^{-}\right)^{p-1} h \mathrm{~d} z \\
-\int_{\Omega} \lambda g\left(z, u_{n}^{+}\right) h \mathrm{~d} z-\int_{\Omega} f\left(z, u_{n}^{+}\right) h \mathrm{~d} z \mid \leq \frac{\epsilon_{n}\|h\|}{1+\left\|u_{n}\right\|} \\
\text { for all } h \in W^{1, p}(\Omega), \text { with } \epsilon_{n} \rightarrow 0^{+} .
\end{gathered}
$$

In (15), we choose $h=-u_{n}^{-} \in W^{1, p}(\Omega)$. Then,

$$
\begin{aligned}
& \gamma_{p}\left(u_{n}^{-}\right)+\eta\left\|u_{n}^{-}\right\|_{p}^{p} \leq \epsilon_{n} \quad \text { for all } n \in \mathbb{N}, \\
\Rightarrow & c_{12}\left\|u_{n}^{-}\right\|^{p} \leq \epsilon_{n} \quad \text { for some } c_{12}>0, \text { all } n \in \mathbb{N}\left(\text { recall that } \eta>\|\xi\|_{\infty}\right), \\
\Rightarrow & u_{n}^{-} \rightarrow 0 \text { in } W^{1, p}(\Omega) \text { as } n \rightarrow \infty .
\end{aligned}
$$

On the other hand, if, in (15), we choose $h=u_{n}^{+} \in W^{1, p}(\Omega)$, then we obtain

$$
-\gamma_{p}\left(u_{n}^{+}\right)+\int_{\Omega}\left[\lambda g\left(z, u_{n}^{+}\right) u_{n}^{+}+f\left(z, u_{n}^{+}\right) u_{n}^{+}\right] \mathrm{d} z \leq \epsilon_{n} \quad \text { for all } n \in \mathbb{N}
$$

From (13) and (16) and from hypothesis $\mathrm{H}(g)$ and $\mathrm{H}(f)(\mathrm{i})$, we have

$$
\gamma_{p}\left(u_{n}^{+}\right)-\int_{\Omega} p\left[\lambda G\left(z, u_{n}^{+}\right)+F\left(z, u_{n}^{+}\right)\right] \mathrm{d} z \leq M_{2} \quad \text { for some } M_{2}>0 \text {, all } n \in \mathbb{N} \text {. }
$$

We add (17) and (18). Then,

$$
\begin{aligned}
& \int_{\Omega} \lambda\left[g\left(z, u_{n}^{+}\right) u_{n}^{+}-p G\left(z, u_{n}^{+}\right)\right] \mathrm{d} z+\int_{\Omega}\left[f\left(z, u_{n}^{+}\right) u_{n}^{+}-p F\left(z, u_{n}^{+}\right)\right] \mathrm{d} z \leq M_{3} \\
& \text { for some } M_{3}>0, \text { all } n \in \mathbb{N}, \\
\Rightarrow & \int_{\Omega}\left[f\left(z, u_{n}^{+}\right) u_{n}^{+}-p F\left(z, u_{n}^{+}\right)\right] \mathrm{d} z \leq \int_{\Omega} \lambda\left[g\left(z, u_{n}^{+}\right) u_{n}^{+}-p G\left(z, u_{n}^{+}\right)\right] \mathrm{d} z+M_{3} \\
& \text { for all } n \in \mathbb{N} .
\end{aligned}
$$

Hypotheses $\mathrm{H}(f)(\mathrm{i})$, (iii) imply that we can find $c_{13}, c_{14}>0$ such that

$$
c_{13} x^{\tau}-c_{14} \leq f(z, x) x-p F(z, x) \quad \text { for a.a. } z \in \Omega \text {, all } x \geq 0 .
$$

In addition, from hypotheses $\mathrm{H}(g)$, we see that we can find $c_{15}, c_{16}>0$ such that

$$
p G(z, x)-g(z, x) x \leq c_{15} x^{q}+c_{16} \quad \text { for a.a. } z \in \Omega \text {, all } x \geq 0 \text {. }
$$

In (19), we use (20) and (21). Since $q<\tau$, we obtain

$$
\begin{aligned}
& \left\|u_{n}\right\|_{\tau}^{\tau} \leq c_{17}\left[1+\left\|u_{n}\right\|_{\tau}^{q}\right] \quad \text { for some } c_{17}>0, \text { all } n \in \mathbb{N}, \\
\Rightarrow \quad & \left\{u_{n}\right\}_{n \geq 1} \subseteq L^{\tau}(\Omega) \text { is bounded. }
\end{aligned}
$$


From hypothesis $\mathrm{H}(f)$ (iii), it is clear that we can have $\tau<r<p^{*}$. Then, let $t \in(0,1)$ be such that

$$
\frac{1}{r}=\frac{1-t}{\tau}+\frac{t}{p^{*}}
$$

Invoking the interpolation inequality (see Papageorgiou-Winkert [23], p. 116), we have

$$
\begin{aligned}
\left\|u_{n}\right\|_{r} & \leq\left\|u_{n}\right\|_{\tau}^{1-t}\left\|u_{n}\right\|_{p^{*}}^{t} \\
\Rightarrow \quad\left\|u_{n}\right\|_{r}^{r} & \leq c_{18}\left\|u_{n}\right\|^{t r} \quad \text { for some } c_{18}>0 \text {, all } n \in \mathbb{N} \text { (see (22)). }
\end{aligned}
$$

Hypotheses $\mathrm{H}(g), \mathrm{H}(f)$ (i) imply that

$$
\lambda g(z, x) x+f(z, x) x \leq c_{19}\left[1+x^{r}\right] \quad \text { for a.a. } z \in \Omega \text {, all } x \geq 0, \text { some } c_{19}>0 .
$$

Recall that

$$
\gamma_{p}\left(u_{n}^{+}\right) \leq \int_{\Omega}\left[\lambda g\left(z, u_{n}^{+}\right) u_{n}^{+}+f\left(z, u_{n}^{+}\right) u_{n}^{+}\right] \mathrm{d} z+c_{20} \quad \text { for some } c_{20}>0 \text {, all } n \in \mathbb{N} .
$$

By assuming that $r>p$ is close to $p^{*}$ (see hypothesis $\mathrm{H}(f)(\mathrm{i})$ ), we can always have that $\tau \geq p$. Then, from (22), we infer that

$$
\left\{u_{n}\right\}_{n \geq 1} \subseteq L^{p}(\Omega) \text { is bounded. }
$$

Hence, with $\eta>\|\xi\|_{\infty}$, we have

$$
\begin{aligned}
& \gamma_{p}\left(u_{n}^{+}\right)+\eta\left\|u_{n}^{+}\right\|_{p}^{p} \leq c_{21}\left[\left\|u_{n}^{+}\right\|_{r}^{r}+1\right] \\
& \text { for some } c_{21}>0 \text {, all } n \in \mathbb{N}(\text { see }(25)-(27)), \\
\Rightarrow \quad & \left\|u_{n}^{+}\right\|^{p} \leq c_{22}\left[\left\|u_{n}^{+}\right\|_{r}^{r}+1\right] \quad \text { for some } c_{22}>0 \text {, all } n \in \mathbb{N}, \\
\Rightarrow & \left\|u_{n}^{+}\right\|^{p} \leq c_{23}\left[\left\|u_{n}^{+}\right\|^{t r}+1\right] \quad \text { for some } c_{23}>0 \text {, all } n \in \mathbb{N} \text { (see (24)). }
\end{aligned}
$$

From the hypothesis on $\tau$ (see $\mathrm{H}(f)$ (iii)) and (23), we infer that $t r<p$. Hence, from (28), it follows that

$$
\begin{aligned}
& \left\{u_{n}^{+}\right\}_{n \geq 1} \subseteq W^{1, p}(\Omega) \text { is bounded, } \\
\Rightarrow \quad & \left\{u_{n}\right\}_{n \geq 1} \subseteq W^{1, p}(\Omega) \text { is bounded (see (16)). }
\end{aligned}
$$

By passing to a suitable subsequence if necessary, we may assume that

$$
u_{n} \stackrel{w}{\rightarrow} u \text { in } W^{1, p}(\Omega) \quad \text { and } \quad u_{n} \rightarrow u \text { in } L^{r}(\Omega) \text { and in } L^{p}(\partial \Omega) .
$$

In (15), we choose $h=u_{n}-u \in W^{1, p}(\Omega)$, pass to the limit as $n \rightarrow \infty$ and use (29). Then,

$$
\begin{aligned}
& \lim _{n \rightarrow \infty}\left\langle A\left(u_{n}\right), u_{n}-u\right\rangle=0 \\
\Rightarrow \quad & u_{n} \rightarrow u \text { in } W^{1, p}(\Omega) \text { (see Proposition } 1 \text { ). }
\end{aligned}
$$

This proves that $\widehat{\varphi}_{\lambda}$ satisfies the C-condition and so we have established the Claim.

Then, (11), (12), and the Claim permit the use of the mountain pass theorem. Thus, there exists $u_{0} \in K_{\widehat{\varphi}_{\lambda}} \backslash\{0\}$ (see (11)). We have

$$
\begin{aligned}
& \left\langle A\left(u_{0}\right), h\right\rangle+\int_{\Omega} \xi(z)\left|u_{0}\right|^{p-2} u_{0} h \mathrm{~d} z+\int_{\partial \Omega} \beta(z)\left|u_{0}\right|^{p-2} u_{0} h \mathrm{~d} \sigma-\int_{\Omega} \eta\left(u_{0}^{-}\right)^{p-1} h \mathrm{~d} z \\
= & \int_{\Omega}\left[\lambda g\left(z, u_{0}^{+}\right)+f\left(z, u_{0}^{+}\right)\right] h \mathrm{~d} z \quad \text { for all } h \in W^{1, p}(\Omega) .
\end{aligned}
$$


We choose $h=-u_{0}^{-} \in W^{1, p}(\Omega)$. Then,

$$
\begin{aligned}
& \gamma_{p}\left(u_{0}^{-}\right)+\eta\left\|u_{0}^{-}\right\|_{p}^{p}=0 \quad \text { (see (2), (3)), } \\
\Rightarrow \quad & \left.u_{0} \geq 0, u_{0} \neq 0 \quad \text { (since } \eta>\|\xi\|_{\infty}\right) .
\end{aligned}
$$

From (30), it follows that

$$
\begin{aligned}
& u_{0} \in \mathscr{S}_{\lambda} \subseteq \operatorname{int} C_{+} \quad \text { for all } \lambda \in\left(0, \lambda_{0}\right) \\
\Rightarrow \quad & \left(0, \lambda_{0}\right) \subseteq \mathscr{L} \neq \varnothing .
\end{aligned}
$$

\subsection{Structural Properties of $\mathscr{L}$}

In this subsection, we show that $\mathscr{L}$ is an interval and establish a kind of monotonicity property for the solution multifunction $\lambda \mapsto \mathscr{S}_{\lambda}$.

Proposition 3. If hypotheses $\mathrm{H}(\xi), \mathrm{H}(\beta), \mathrm{H}(g), \mathrm{H}(f), \mathrm{H}_{0}$ hold, $\lambda \in \mathscr{L}$ and $0<\mu<\lambda$, then $\mu \in \mathscr{L}$.

Proof. Since $\lambda \in \mathscr{L}$, we can find $u_{\lambda} \in \mathscr{S}_{\lambda} \subseteq \operatorname{int} C_{+}$. Using $u_{\lambda}$, we consider the following truncation-perturbation of the reaction on problem $\left(P_{\mu}\right)$ :

$$
\begin{aligned}
& k_{\mu}(z, x)= \begin{cases}\mu g\left(z, x^{+}\right)+f\left(z, x^{+}\right)+\eta\left(x^{+}\right)^{p-1} & \text { if } x \leq u_{\lambda}(z) \\
\mu g\left(z, u_{\lambda}(z)\right)+f\left(z, u_{\lambda}(z)\right)+\eta u_{\lambda}(z)^{p-1} & \text { if } u_{\lambda}(z)<x\end{cases} \\
& \text { (recall that } \left.\eta>\|\xi\|_{\infty}\right)
\end{aligned}
$$

This is a Carathéodory function. Let $K_{\mu}(z, x)=\int_{0}^{x} k_{\mu}(z, s) \mathrm{d} s$. We introduce the $C^{1}$-functional $\widehat{\psi}_{\mu}: W^{1, p}(\Omega) \rightarrow \mathbb{R}$ defined by

$$
\widehat{\psi}_{\mu}(u)=\frac{1}{p} \gamma_{p}(u)+\frac{\eta}{p}\|u\|_{p}^{p}-\int_{\Omega} K_{\mu}(z, u) \mathrm{d} z \quad \text { for all } u \in W^{1, p}(\Omega) .
$$

From (31) and, since $\eta>\|\xi\|_{\infty}$, we see that

$$
\widehat{\psi}_{\mu}(\cdot) \text { is coercive. }
$$

Moreover, using the Sobolev embedding theorem and the compactness of the trace map, we have that

$$
\widehat{\psi}_{\mu}(\cdot) \text { is sequentially weakly lower semicontinuous. }
$$

Then, the Weierstrass-Tonelli theorem provides $u_{\mu} \in W^{1, p}(\Omega)$ such that

$$
\widehat{\psi}_{\mu}\left(u_{\mu}\right)=\inf \left[\widehat{\psi}_{\mu}(u): u \in W^{1, p}(\Omega)\right] .
$$

Hypothesis $\mathrm{H}(f)$ (iv) implies that, given $\epsilon>0$, we can find $\delta \in\left(0, \min \left\{\delta_{0}, \min _{\bar{\Omega}} u_{\lambda}\right\}\right)$ (see hypothesis $\mathrm{H}(g)$ (iii) and recall that $u_{\lambda} \in \operatorname{int} C_{+}$) such that

$$
-\frac{\epsilon}{q} x^{q} \leq F(z, x) \text { for a.a. } z \in \Omega \text {, all } 0 \leq x \leq \delta .
$$


In addition, from hypothesis $\mathrm{H}(\mathrm{g})$ (iii), we have

$$
\frac{c_{0}}{q} x^{q} \leq G(z, x) \text { for a.a. } z \in \Omega \text {, all } 0 \leq x \leq \delta_{0} .
$$

Let $\widehat{\lambda}_{1} \in \mathbb{R}$ be the first eigenvalue of the operator $u \mapsto-\Delta_{p} u+\xi(z) u$ with the Robin boundary condition and let $\widehat{u}_{1} \in W^{1, p}(\Omega)$ be the $L^{p}$-normalized (that is, $\left\|\widehat{u}_{1}\right\|_{p}=1$ ) eigenfunction corresponding to $\widehat{\lambda}_{1}$. We know that $\widehat{u}_{1}$ has a fixed sign and we can take it to be positive. The nonlinear regularity theory and the nonlinear Hopf boundary point theorem imply that $\widehat{u}_{1} \in \operatorname{int} C_{+}$. We have $\gamma_{p}\left(\widehat{u}_{1}\right)=\widehat{\lambda}_{1}$ (for details, we refer to Papageorgiou-Rădulescu [24]). Let $t \in(0,1)$ be small such that

$$
t \widehat{u}_{1}(z) \in(0, \delta] \text { for a.a. } z \in \bar{\Omega} \text {. }
$$

We have

$$
\widehat{\psi}_{\mu}\left(t \widehat{u}_{1}\right) \leq \frac{t^{p}}{p} \widehat{\lambda}_{1}-\frac{t^{q}}{q}\left[c_{0}-\epsilon\right]\left\|\widehat{u}_{1}\right\|_{q}^{q} \quad \text { (see (33)-(35), (31)). }
$$

Since $q<p$, choosing $t \in(0,1)$ even smaller if necessary, we have

$$
\begin{aligned}
& \widehat{\psi}_{\mu}\left(t \widehat{u}_{1}\right)<0, \\
\Rightarrow & \widehat{\psi}_{\mu}\left(u_{\mu}\right)<0=\widehat{\psi}_{\mu}(0) \quad(\text { see }(32)), \\
\Rightarrow & u_{\mu} \neq 0 .
\end{aligned}
$$

From (32), we have

$$
\begin{gathered}
\widehat{\psi}_{\mu}^{\prime}\left(u_{\mu}\right)=0 \\
\Rightarrow\left\langle A\left(u_{\mu}\right), h\right\rangle+\int_{\Omega}[\xi(z)+\eta]\left|u_{\mu}\right|^{p-2} u_{\mu} h \mathrm{~d} z+\int_{\partial \Omega} \beta(z)\left|u_{\mu}\right|^{p-2} u_{\mu} h \mathrm{~d} \infty \\
=\int_{\Omega} k_{\mu}\left(z, u_{\mu}\right) h \mathrm{~d} z \quad \text { for all } h \in W^{1, p}(\Omega) .
\end{gathered}
$$

In (36), first we choose $h=-u_{\mu}^{-} \in W^{1, p}(\Omega)$. Then

$$
\begin{aligned}
& \gamma_{p}\left(u_{\mu}^{-}\right)+\eta\left\|u_{\mu}^{-}\right\|_{p}^{p}=0 \quad \text { (see (31)), } \\
\Rightarrow \quad & \left.u_{\mu} \geq 0, u_{\mu} \neq 0 \quad \text { (since } \eta>\|\xi\|_{\infty}\right) .
\end{aligned}
$$

Next, in (36), we choose $h=\left(u_{\mu}-u_{\lambda}\right)^{+} \in W^{1, p}(\Omega)$. Then,

$$
\begin{gathered}
\left\langle A\left(u_{\mu}\right),\left(u_{\mu}-u_{\lambda}\right)^{+}\right\rangle+\int_{\Omega}[\xi(z)+\eta] u_{\mu}^{p-1}\left(u_{\mu}-u_{\lambda}\right)^{+} \mathrm{d} z+\int_{\partial \Omega} \beta(z) u_{\mu}^{p-1}\left(u_{\mu}-u_{\lambda}\right)^{+} \mathrm{d} \sigma \\
=\int_{\Omega}\left[\mu g\left(z, u_{\lambda}\right)+f\left(z, u_{\lambda}\right)+\eta u_{\lambda}^{p-1}\right]\left(u_{\mu}-u_{\lambda}\right)^{+} \mathrm{d} z \quad \text { (see (31)) } \\
\left.\leq \int_{\Omega}\left[\lambda g\left(z, u_{\lambda}\right)+f\left(z, u_{\lambda}\right)+\eta u_{\lambda}^{p-1}\right]\left(u_{\mu}-u_{\lambda}\right)^{+} \mathrm{d} z \quad \text { (since } \lambda>\mu, g \geq 0\right) \\
=\left\langle A\left(u_{\lambda}\right),\left(u_{\mu}-u_{\lambda}\right)^{+}\right\rangle+\int_{\Omega}[\xi(z)+\eta] u_{\lambda}^{p-1}\left(u_{\mu}-u_{\lambda}\right)^{+} \mathrm{d} z+\int_{\partial \Omega} \beta(z) u_{\lambda}^{p-1}\left(u_{\mu}-u_{\lambda}\right)^{+} \mathrm{d} \sigma \\
\quad\left(\text { since } u_{\lambda} \in \mathscr{S}_{\lambda}\right) \quad \\
\Rightarrow u_{\mu} \leq u_{\lambda} .
\end{gathered}
$$

Thus, we have proved that

$$
u_{\mu} \in\left[0, u_{\lambda}\right], u_{\mu} \neq 0 .
$$


From (37), (31) and (36), we infer that

$$
\begin{aligned}
& u_{\mu} \in \mathscr{S}_{\mu} \subseteq \operatorname{int} C_{+}, \\
\Rightarrow & \mu \in \mathscr{L} .
\end{aligned}
$$

An interesting byproduct of the above proof, is the following "monotonicity" result for the solution set multifunction $\lambda \mapsto \mathscr{S}_{\lambda}$.

Corollary 1. If hypotheses $\mathrm{H}(\xi), \mathrm{H}(\beta), \mathrm{H}(g), \mathrm{H}(f), \mathrm{H}_{0}$ hold, $\lambda \in \mathscr{L}, u_{\lambda} \in \mathscr{S}_{\lambda} \subseteq$ int $C_{+}$and $0<\mu<\lambda$, then $\mu \in \mathscr{L}$ and we can find $u_{\mu} \in \mathscr{S}_{\mu} \subseteq$ int $C_{+}$such that $u_{\mu} \leq u_{\lambda}$.

In fact, we can improve the conclusion of this Corollary.

Proposition 4. If hypotheses $\mathrm{H}(\xi), \mathrm{H}(\beta), \mathrm{H}(g), \mathrm{H}(f), \mathrm{H}_{0}$ hold, $\lambda \in \mathscr{L}, u_{\lambda} \in \mathscr{S}_{\lambda} \subseteq$ int $C_{+}$and $0<\mu<\lambda$, then $\mu \in \mathscr{L}$ and we can find $u_{\mu} \in \mathscr{S}_{\mu} \subseteq$ int $C_{+}$such that $u_{\lambda}-u_{\mu} \in \widehat{K}_{+}$.

Proof. From Corollary 1, we already know that $\mu \in \mathscr{L}$ and that we can find $u_{\mu} \in \mathscr{S}_{\mu} \subseteq \operatorname{int} C_{+}$ such that

$$
0 \leq u_{\mu} \leq u_{\lambda}
$$

Let $\rho=\left\|u_{\lambda}\right\|_{\infty}, B=[\mu, \lambda]$ and let $\widehat{\xi}_{\rho}^{B}>0$ be as postulated by hypothesis $\mathrm{H}_{0}$. Clearly, we can always take $\widehat{\zeta}_{\rho}^{B}>\|\xi\|_{\infty}$. We have

$$
\begin{aligned}
-\Delta_{p} & u_{\mu}(z)+\left[\xi(z)+\widehat{\xi}_{\rho}^{B}\right] u_{\mu}(z)^{p-1} \\
& =\mu g\left(z, u_{\mu}(z)\right)+f\left(z, u_{\mu}(z)\right)+\widehat{\xi}_{\rho}^{B} u_{\mu}(z)^{p-1} \\
& =\lambda g\left(z, u_{\mu}(z)\right)+f\left(z, u_{\mu}(z)\right)+\widehat{\xi}_{\rho}^{B} u_{\mu}(z)^{p-1}-[\lambda-\mu] g\left(z, u_{\mu}(z)\right) \\
& \left.\leq \lambda g\left(z, u_{\lambda}(z)\right)+f\left(z, u_{\lambda}(z)\right)+\widehat{\xi}_{\rho}^{B} u_{\lambda}(z)^{p-1} \quad \text { (see }(38), \mathrm{H}_{0} \text { and recall that } \mu<\lambda\right) \\
& =-\Delta_{p} u_{\lambda}(z)+\left[\xi(z)+\widehat{\xi}_{\rho}^{B}\right] u_{\lambda}(z)^{p-1} \quad \text { for a.a. } z \in \Omega .
\end{aligned}
$$

Let $s=\min _{\bar{\Omega}} u_{\mu}>0$ (recall that $u_{\mu} \in \operatorname{int} C_{+}$). Then, using hypothesis $\mathrm{H}(g)$ (iii), we have

$$
(\lambda-\mu) g\left(z, u_{\mu}(z)\right) \geq(\lambda-\mu) m_{s}>0
$$

Then, from (39) and Proposition 2.10 of Papageorgiou-Rădulescu-Repovš [25], we infer that $u_{\lambda}-u_{\mu} \in \widehat{K}_{+}$.

\subsection{The Critical Parameter $\lambda^{*}$}

Here, we show that $\lambda^{*}<+\infty$, that is, the set of admissible parameters $\mathscr{L}$ is a bounded interval and also we show that $\lambda^{*}>0$ is admissible, that is, $\lambda^{*} \in \mathscr{L}$. To this end, first, we prove a weak form of the antimaximum principle for the Robin $p$-Laplacian plus and indefinite potential (see Godoy-Gossez-Paczka [26]). We start with a lemma which is stated in a more general form than the one that we need in the sequel, but which is of independent interest.

Lemma 1. If $u, \vartheta \in W^{1, p}(\Omega) \cap C^{1}(\Omega) \cap L^{\infty}(\Omega), u(z)>0$ and $\vartheta(z) \geq 0$ for all $z \in \Omega$, then we can find $c^{*}>0$ independent of $u$ such that

$$
\int_{\Omega}|D u|^{p-2}\left(D u, D\left(\frac{\vartheta^{p}}{u^{p-1}}\right)\right)_{\mathbb{R}^{N}} \mathrm{~d} z \leq c^{*}\|D \vartheta\|_{p}^{p} .
$$


Proof. For $\epsilon>0$, let $u_{\epsilon}=u+\epsilon$. We have

$$
\begin{aligned}
& |D u|^{p-2}\left(D u, D\left(\frac{\vartheta^{p}}{u_{\epsilon}^{p-1}}\right)\right)_{\mathbb{R}^{N}} \\
= & |D u|^{p-2}\left(D u, \frac{\vartheta^{p-1}\left(p u_{\epsilon} D \vartheta-(p-1) \vartheta D u\right)}{u_{\epsilon}^{p}}\right)_{\mathbb{R}^{N}} \\
= & p\left(\frac{\vartheta}{u_{\epsilon}}\right)^{p-1}|D u|^{p-2}(D u, D \vartheta)_{\mathbb{R}^{N}}-(p-1)\left(\frac{\vartheta}{u_{\epsilon}}\right)^{p}|D u|^{p} \\
\leq & p\left(\frac{\vartheta}{u_{\epsilon}}\right)^{p-1}|D u|^{p-1}|D \vartheta|-(p-1)\left(\frac{\vartheta}{u_{\epsilon}}\right)^{p}|D u|^{p}
\end{aligned}
$$

Using Young's inequality with $\delta>0$ (see Papageorgiou-Winkert [23], p. 113), we have

$$
p\left(\frac{\vartheta}{u_{\epsilon}}\right)^{p-1}|D u|^{p-1}|D \vartheta| \leq \frac{1}{\delta p}|D \vartheta|^{p}+\delta(p-1)\left(\frac{\vartheta}{u_{\epsilon}}\right)^{p}|D u|^{p} .
$$

Returning to (40) and using (41), we obtain

$$
|D u|^{p-2}\left(D u, D\left(\frac{\vartheta^{p}}{u_{\epsilon}^{p-1}}\right)\right)_{\mathbb{R}^{N}} \leq \frac{1}{\delta p}|D \vartheta|^{p}-[1-\delta](p-1)\left(\frac{\vartheta}{u_{\epsilon}}\right)^{p-1}|D u|^{p} .
$$

Choosing $\delta \in(0,1)$, we obtain

$$
|D u|^{p-2}\left(D u, D\left(\frac{\vartheta^{p}}{u_{\epsilon}^{p-1}}\right)\right)_{\mathbb{R}^{N}} \leq c^{*}|D \vartheta|^{p} \quad \text { with } c^{*}=\frac{1}{\delta p}>0 .
$$

Note that $\frac{\vartheta^{p}}{u_{\epsilon}^{p-1}} \in W^{1, p}(\Omega)$. In addition, $u_{\epsilon} \in \operatorname{int} C_{+}$. Thus, according to Proposition 4.1.22 of Papageorgiou-Rădulescu-Repovš [18], we can find $\mu>0$ such that $\vartheta \leq \mu u_{\epsilon}$. Then, we have

$$
0 \leq \frac{\vartheta^{p}}{u_{\epsilon}^{p-1}} \leq\|\vartheta\|_{\infty}\left(\frac{\mu u_{\epsilon}}{u_{\epsilon}}\right)^{p-1}=\mu^{p-1}\|\vartheta\|_{\infty}
$$

In addition,

$$
\begin{aligned}
\left|D\left(\frac{\vartheta^{p}}{u_{\epsilon}^{p-1}}\right)\right| & \leq \frac{p u_{\epsilon} \vartheta^{p-1}|D \vartheta|+(p-1) \vartheta^{p}|D u|}{u_{\epsilon}^{p}} \\
& \leq \frac{u_{\epsilon}^{p}[p|D \vartheta|+(p-1)|D u|]}{u_{\epsilon}^{p}} \\
& =p|D \vartheta|+(p-1)|D u| \in L^{p}(\Omega) .
\end{aligned}
$$

Therefore, $\left\{\frac{\vartheta^{p}}{u_{\epsilon}^{p-1}}\right\}_{\epsilon>0} \subseteq W^{1, p}(\Omega)$ is bounded. Hence, we have

$$
\frac{\vartheta^{p}}{u_{\epsilon}^{p-1}} \stackrel{w}{\rightarrow} \frac{\vartheta^{p}}{u^{p-1}} \quad \text { in } W^{1, p}(\Omega) \text { as } \epsilon \rightarrow 0^{+} .
$$

From (42), we have

$$
\begin{aligned}
\left\langle A(u), \frac{\vartheta^{p}}{u_{\epsilon}^{p-1}}\right\rangle=\int_{\Omega}|D u|^{p-2}\left(D u, D\left(\frac{\vartheta^{p}}{u_{\epsilon}^{p-1}}\right)\right)_{\mathbb{R}^{N}} \mathrm{~d} z \leq c^{*}\|D \vartheta\|_{p}^{p} \quad \text { for all } \epsilon>0 \\
\Rightarrow\left\langle A(u), \frac{\vartheta^{p}}{u^{p-1}}\right\rangle=\int_{\Omega}|D u|^{p-2}\left(D u, D\left(\frac{\vartheta^{p}}{u^{p-1}}\right)\right)_{\mathbb{R}^{N}} \mathrm{~d} z \leq c^{*}\|D \vartheta\|_{p}^{p} \quad \text { (see (43)). }
\end{aligned}
$$


Let $e \in L^{\infty}(\Omega), e(z) \geq 0$ for a.a. $z \in \Omega, e \neq 0$. We consider the nonlinear Robin problem

$$
\begin{cases}-\Delta_{p} u(z)+\xi(z) u(z)^{p-1}=\lambda u(z)^{p-1}+e(z) & \text { in } \Omega \\ \frac{\partial u}{\partial n_{p}}+\beta(z) u^{p-1}=0 & \text { on } \partial \Omega \\ u>0 & \end{cases}
$$

We set $\widetilde{\xi}=\frac{1}{c^{*}} \xi, \widetilde{\beta}=\frac{1}{c^{*}} \beta$ and let $\widetilde{\lambda}_{1} \in \mathbb{R}$ the principal eigenvalue for the operator $u \mapsto-\Delta_{p} u+$ $\xi(z)|u|^{p-2} u$ with the Robin boundary condition $\frac{\partial u}{\partial n_{p}}+\widetilde{\beta}(z)|u|^{p-2} u=0$ on $\partial \Omega$ (see [24]).

We have the following weak antimaximum principle for problem (44).

Proposition 5. If $\lambda>\widetilde{\lambda}_{1} c^{*}$, then problem (44) has no solution.

Proof. Arguing by contradiction, suppose that problem (44) has a positive solution $u$. As before, the nonlinear regularity theory and the nonlinear Hopf boundary point theorem imply that $u \in \operatorname{int} C_{+}$.

Let $\vartheta \in W^{1, p}(\Omega) \cap C^{1}(\Omega) \cap L^{\infty}(\Omega), \vartheta \geq 0$. From Lemma 1 and using the nonlinear Green's identity (see Gasiński-Papageorgiou [1], p. 211), we have

$$
\begin{aligned}
c^{*}\|D \vartheta\|_{p}^{p} & \geq \int_{\Omega}|D u|^{p-2}\left(D u, D\left(\frac{\vartheta^{p}}{u^{p-1}}\right)\right)_{\mathbb{R}^{N}} \mathrm{~d} z \\
& =\int_{\Omega}\left(-\Delta_{p} u\right) \frac{\vartheta^{p}}{u^{p-1}} \mathrm{~d} z+\int_{\partial \Omega}\left(\frac{\partial u}{\partial n_{p}}\right) \frac{\vartheta^{p}}{u^{p-1}} \mathrm{~d} \sigma \\
& \geq \int_{\Omega}[\lambda-\xi(z)] u^{p-1}\left(\frac{\vartheta^{p}}{u^{p-1}}\right) \mathrm{d} z-\int_{\partial \Omega} \beta(z) u^{p-1} \frac{\vartheta^{p}}{u^{p-1}} \mathrm{~d} \sigma \\
& =\int_{\Omega}[\lambda-\xi(z)] \vartheta^{p} \mathrm{~d} z-\int_{\partial \Omega} \beta(z) \vartheta^{p} \mathrm{~d} \sigma .
\end{aligned}
$$

If $\widetilde{\gamma}_{p}(y)=\|D y\|_{p}^{p}+\int_{\Omega} \widetilde{\zeta}(z)|y|^{p} \mathrm{~d} z+\int_{\partial \Omega} \widetilde{\beta}(z)|y|^{p} \mathrm{~d} \sigma$ for all $y \in W^{1, p}(\Omega)$, then, from (45), we have

$$
\widetilde{\gamma}_{p}(\vartheta) \geq \frac{\lambda}{c^{*}}\|\vartheta\|_{p}^{p} \quad \text { for all } \vartheta \in W^{1, p}(\Omega) \cap C^{1}(\Omega) \cap L^{\infty}(\Omega), \vartheta \geq 0
$$

By density, this inequality still holds for all $\vartheta \in W^{1, p}(\Omega)$. However, then, from (46), we have

$$
\widetilde{\lambda}_{1} \geq \frac{\lambda}{c^{*}}>\widetilde{\lambda}_{1}
$$

a contradiction. This proves that, for $\lambda>\widetilde{\lambda}_{1} c^{*}$, problem (44) has no solution.

Using this weak antimaximum principle, we can show that $\lambda^{*}<\infty$.

Proposition 6. If hypotheses $\mathrm{H}(\xi), \mathrm{H}(\beta), \mathrm{H}(g), \mathrm{H}(f), \mathrm{H}_{0}$ hold, then $\lambda^{*}<\infty$.

Proof. Let $\mu>\widetilde{\lambda}_{1} c^{*}$. On account of hypotheses $\mathrm{H}(f)$ (ii), (iii), we have that

$$
\lim _{x \rightarrow+\infty} \frac{f(z, x)}{x^{p-1}}=+\infty \quad \text { uniformly for a.a. } z \in \Omega .
$$

Thus, we can find $M>0$ such that

$$
f(z, x) \geq \mu x^{p-1} \text { for a.a. } z \in \Omega \text {, all } x \geq M .
$$


In addition, hypothesis $\mathrm{H}(\mathrm{g})$ (iii) implies that

$$
\lim _{x \rightarrow 0^{+}} \frac{g(z, x)}{x^{p-1}}=+\infty \quad \text { uniformly for a.a. } z \in \Omega .
$$

Thus, for every $\lambda>0$, we can find $\delta_{\lambda} \in\left(0, \delta_{0}\right]$ (see hypothesis $\mathrm{H}(g)$ (iii)) such that

$$
\lambda g(z, x) \geq \mu x^{p-1} \quad \text { for a.a. } z \in \Omega, \text { all } 0 \leq x \leq \delta_{\lambda} .
$$

We fix $\lambda_{0}>0$. Using hypothesis $\mathrm{H}(\mathrm{g})$ (iv), we see that we can find $\bar{\lambda}>\lambda_{0}$ such that

$$
\bar{\lambda} g(z, x) \geq \mu M^{p-1} \geq \mu x^{p-1} \quad \text { for a.a. } z \in \Omega \text {, all } x \in\left[\delta_{\lambda_{0}}, M\right] .
$$

From (47)-(49) and since $g \geq 0, f \geq 0$, we infer that

$$
\bar{\lambda} g(z, x)+f(z, x) \geq \mu x^{p-1} \quad \text { for a.a. } z \in \Omega \text {, all } x \geq 0 .
$$

Let $\lambda>\bar{\lambda}$ and suppose that $\lambda \in \mathscr{L}$. Then, we can find $u_{\lambda} \in \mathscr{S}_{\lambda} \subseteq \operatorname{int} C_{+}$and we have

$$
\begin{aligned}
-\Delta_{p} & u_{\lambda}(z)+\xi(z) u_{\lambda}(z)^{p-1} \\
& =\lambda g\left(z, u_{\lambda}(z)\right)+f\left(z, u_{\lambda}(z)\right) \\
& =\bar{\lambda} g\left(z, u_{\lambda}(z)\right)+f\left(z, u_{\lambda}(z)\right)+(\lambda-\bar{\lambda}) g\left(z, u_{\lambda}(z)\right) .
\end{aligned}
$$

We set $e(z)=(\lambda-\bar{\lambda}) g\left(z, u_{\lambda}(z)\right)$. Then, $e \in L^{\infty}(\Omega), e(z) \geq 0$ for a.a. $z \in \Omega, e \not \equiv 0$ (recall that $\lambda>\bar{\lambda}, u_{\lambda} \in \operatorname{int} C_{+}$and use hypothesis $\mathrm{H}(g)$ (iii)). We introduce the Carathéodory function

$$
\widehat{l}(z, x)=\left\{\begin{array}{ll}
(\mu+\eta)\left(x^{+}\right)^{p-1}+e(z) & \text { if } x \leq u_{\lambda}(z) \\
(\mu+\eta) u_{\lambda}(z)^{p-1}+e(z) & \text { if } u_{\lambda}(z)<x
\end{array} \quad \text { (recall that } \eta>\|\xi\|_{\infty}\right) .
$$

We set $\widehat{L}(z, x)=\int_{0}^{x} \widehat{l}(z, s)$ ds and consider the $C^{1}$-functional $\widehat{\vartheta}: W^{1, p}(\Omega) \rightarrow \mathbb{R}$ defined by

$$
\widehat{\vartheta}(u)=\frac{1}{p} \gamma_{p}(u)+\frac{\eta}{p}\|u\|_{p}^{p}-\int_{\Omega} \widehat{L}(z, u) \mathrm{d} z \quad \text { for all } u \in W^{1, p}(\Omega) .
$$

From (51) and since $\eta>\|\xi\|_{\infty}$, we see that $\widehat{\vartheta}(\cdot)$ is coercive. In addition, it is sequentially weakly lower semicontinuous. Thus, we can find $\widetilde{u} \in W^{1, p}(\Omega)$ such that

$$
\widehat{\vartheta}(\widetilde{u})=\inf \left[\widehat{\vartheta}(u): u \in W^{1, p}(\Omega)\right] .
$$

Let $\widetilde{u}_{1} \in \operatorname{int} C_{+}$be the positive, $L^{p}$-normalized eigenfunction corresponding to the principal eigenvalue $\widetilde{\lambda}_{1}$. Let $t \in(0,1)$ be small such that

$$
\left.t \widetilde{u}_{1} \leq u_{\lambda} \quad \text { (recall that } u_{\lambda} \in \operatorname{int} C_{+}\right) .
$$

We can always assume that $c^{*} \geq 1$ (see Lemma 1 ). Then,

$$
\begin{aligned}
\widehat{\vartheta}\left(t \widetilde{u}_{1}\right) & =\frac{t^{p}}{p}\left[\widetilde{\lambda}_{1} c^{*}-\mu\right]-t \int_{\Omega} e \widetilde{u}_{1} \mathrm{~d} z \quad\left(\text { see }(51) \text { and recall that }\left\|\widetilde{u}_{1}\right\|_{p}=1\right) \\
& \leq \frac{t^{p}}{p}\left[\widetilde{\lambda}_{1} c^{*}-\mu\right] \quad\left(\text { since } e, \widetilde{u}_{1} \geq 0\right) .
\end{aligned}
$$


Then, choosing $\mu>\widetilde{\lambda}_{1} c^{*}$ as big, we will have

$$
\begin{aligned}
& \widehat{\vartheta}\left(t \widetilde{u}_{1}\right)<-\|e\|_{1}=\widehat{\vartheta}(0), \\
\Rightarrow & \widehat{\vartheta}(\widetilde{u})<-\|e\|_{1}=\widehat{\vartheta}(0) \quad(\text { see }(52)), \\
\Rightarrow & \widetilde{u} \neq 0 .
\end{aligned}
$$

From (52), we have

$$
\begin{gathered}
\widehat{\vartheta}^{\prime}(\widetilde{u})=0 \\
\Rightarrow \quad\langle A(\widetilde{u}), h\rangle+\int_{\Omega}[\xi(z)+\eta]|\widetilde{u}|^{p-2} \widetilde{u} h \mathrm{~d} z+\int_{\partial \Omega} \beta(z)|\widetilde{u}|^{p-2} \widetilde{u} h \mathrm{~d} \sigma \\
=\int_{\Omega} \widehat{l}(z, \widetilde{u}) h \mathrm{~d} z \quad \text { for all } h \in W^{1, p}(\Omega) .
\end{gathered}
$$

In (54), first we choose $h=-\widetilde{u}^{-} \in W^{1, p}(\Omega)$. Then,

$$
\begin{aligned}
& \gamma_{p}\left(\widetilde{u}^{-}\right)+\eta\left\|\widetilde{u}^{-}\right\|_{p}^{p} \leq 0 \quad(\text { see }(51) \text { and recall that } e \geq 0), \\
\Rightarrow \quad & \widetilde{u} \geq 0, \widetilde{u} \neq 0 \quad\left(\text { since } \eta>\|\xi\|_{\infty}\right) .
\end{aligned}
$$

Next, in (54), we choose $h=\left(\widetilde{u}-u_{\lambda}\right)^{+} \in W^{1, p}(\Omega)$. Then,

$$
\begin{aligned}
& \left\langle A(\widetilde{u}),\left(\widetilde{u}-u_{\lambda}\right)^{+}\right\rangle+\int_{\Omega}[\tilde{\xi}(z)+\eta] \widetilde{u}^{p-1}\left(\widetilde{u}-u_{\lambda}\right)^{+} \mathrm{d} z+\int_{\partial \Omega} \beta(z) \widetilde{u}^{p-1}\left(\widetilde{u}-u_{\lambda}\right)^{+} \mathrm{d} \sigma \\
& =\int_{\Omega}\left([\mu+\eta] u_{\lambda}^{p-1}+e(z)\right)\left(\widetilde{u}-u_{\lambda}\right)^{+} \mathrm{d} z \\
& \leq \int_{\Omega}\left[\lambda g\left(z, u_{\lambda}\right)+f\left(z, u_{\lambda}\right)+\eta u_{\lambda}^{p-1}\right]\left(\widetilde{u}-u_{\lambda}\right)^{+} \mathrm{d} z \quad(\text { see }(50)) \\
& =\left\langle A\left(u_{\lambda}\right),\left(\widetilde{u}-u_{\lambda}\right)^{+}\right\rangle+\int_{\Omega}[\xi(z)+\eta] u_{\lambda}^{p-1}\left(\widetilde{u}-u_{\lambda}\right)^{+} \mathrm{d} z+\int_{\partial \Omega} \beta(z) u_{\lambda}^{p-1}\left(\widetilde{u}-u_{\lambda}\right)^{+} \mathrm{d} \sigma \\
& \left.\quad\left(\text { since } u_{\lambda} \in \mathscr{S}_{\lambda}\right), \quad \Rightarrow \widetilde{u} \leq u_{\lambda} \quad \text { (since } \eta>\|\xi\|_{\infty}\right) .
\end{aligned}
$$

Thus, we have proved that

$$
\widetilde{u} \in\left[0, u_{\lambda}\right], \widetilde{u} \neq 0 .
$$

Then, (51), (54), (55) and [24], imply that

$$
\begin{aligned}
& -\Delta_{p} \widetilde{u}(z)+\xi(z) \widetilde{u}(z)^{p-1}=\mu \widetilde{u}(z)^{p-1}+e(z) \quad \text { for a.a. } z \in \Omega \\
& \frac{\partial \widetilde{u}}{\partial n_{p}}+\beta(z) \widetilde{u}^{p-1}=0 \text { on } \partial \Omega \\
\Rightarrow & \widetilde{u} \in \operatorname{int} C_{+} .
\end{aligned}
$$

However, since $\mu>\widetilde{\lambda}_{1} c^{*}$, we contradict Proposition 5 (the weak antimaximum principle). $\left(0, \lambda^{*}\right]$

Next, we show that the critical parameter $\lambda^{*}>0$ is admissible (that is, $\lambda^{*} \in \mathscr{L}$ ) and so $\mathscr{L}=$

Proposition 7. If hypotheses $\mathrm{H}(\xi), \mathrm{H}(\beta), \mathrm{H}(g), \mathrm{H}(f), \mathrm{H}_{0}$ hold, then $\lambda^{*} \in \mathscr{L}$. 
Proof. For $\lambda>0$ let $\varphi_{\lambda}: W^{1, p}(\Omega) \rightarrow \mathbb{R}$ be the energy functional for problem (1) defined by

$$
\varphi_{\lambda}(u)=\frac{1}{p} \gamma_{p}(u)-\lambda \int_{\Omega} G(z, u) \mathrm{d} z-\int_{\Omega} F(z, u) \mathrm{d} z \quad \text { for all } u \in W^{1, p}(\Omega) .
$$

Consider $\left\{\lambda_{n}\right\}_{n \geq 1} \subseteq\left(0, \lambda^{*}\right)$ such that $\lambda_{n} \uparrow \lambda^{*}$. From the proof of Proposition 3, we see that we can find $u_{n} \in \mathscr{S}_{\lambda_{n}} \subseteq \operatorname{int} C_{+}, n \in \mathbb{N}$, such that

$$
\varphi_{\lambda_{n}}\left(u_{n}\right)<0 \text { for all } n \in \mathbb{N} .
$$

We have

$$
\begin{aligned}
& \left\langle A\left(u_{n}\right), h\right\rangle+\int_{\Omega} \xi(z) u_{n}^{p-1} h \mathrm{~d} z+\int_{\partial \Omega} \beta(z) u_{n}^{p-1} h \mathrm{~d} \sigma=\int_{\Omega}\left[\lambda_{n} g\left(z, u_{n}\right)+f\left(z, u_{n}\right)\right] h \mathrm{~d} z \\
& \text { for all } h \in W^{1, p}(\Omega) \text {, all } n \in \mathbb{N} .
\end{aligned}
$$

Using (56) and (57) and reasoning as in the proof of Proposition 2 (see the proof of the Claim), we show that

$$
\left\{u_{n}\right\}_{n \geq 1} \subseteq W^{1, p}(\Omega) \text { is bounded. }
$$

Thus, we may assume that

$$
u_{n} \stackrel{w}{\rightarrow} u_{*} \text { in } W^{1, p}(\Omega) \quad \text { and } \quad u_{n} \rightarrow u_{*} \text { in } L^{r}(\Omega) \text { and in } L^{p}(\partial \Omega) .
$$

In (57), we choose $h=u_{n}-u_{*} \in W^{1, p}(\Omega)$, pass to the limit as $n \rightarrow \infty$ and use (58). Then,

$$
\begin{aligned}
& \lim _{n \rightarrow \infty}\left\langle A\left(u_{n}\right), u_{n}-u_{*}\right\rangle=0, \\
\Rightarrow \quad & u_{n} \rightarrow u_{*} \text { in } W^{1, p}(\Omega) \text { (see Proposition 1). }
\end{aligned}
$$

We show that $u_{*} \neq 0$. On account of hypotheses $\mathrm{H}(g)$ (iii) and $\mathrm{H}(f)(\mathrm{i})$, we can find $c_{24}>0$ such that

$$
\lambda_{n} g(z, x)+f(z, x) \geq \lambda_{1} c_{0} x^{q-1}-c_{24} x^{r-1} \quad \text { for a.a. } z \in \Omega \text {, all } x \geq 0 \text {, all } n \in \mathbb{N} .
$$

Based on (60), we introduce the following auxiliary Robin problem:

$$
\begin{cases}-\Delta_{p} u(z)+\xi(z) u(z)^{p-1}=\lambda_{1} c_{0} u(z)^{q-1}-c_{24} u(z)^{r-1} & \text { in } \Omega \\ \frac{\partial u}{\partial n_{p}}+\beta(z) u^{p-1}=0 & \text { on } \partial \Omega \\ u>0 & \end{cases}
$$

Let $d: W^{1, p}(\Omega) \rightarrow \mathbb{R}$ be the $C^{1}$-functional defined by

$d(u)=\frac{1}{p} \gamma_{p}(u)+\frac{\eta}{p}\left\|u^{-}\right\|_{p}^{p}+\frac{c_{24}}{r}\left\|u^{+}\right\|_{r}^{r}-\frac{\lambda_{1} c_{0}}{q}\left\|u^{+}\right\|_{q}^{q} \quad$ for all $u \in W^{1, p}(\Omega)$, with $\eta>\|\xi\|_{\infty}$. 
We have

$$
\begin{aligned}
& d(u) \geq c_{25}\left\|u^{-}\right\|^{p}+\frac{1}{p} \gamma_{p}\left(u^{+}\right)+\frac{c_{24}}{r}\left\|u^{+}\right\|_{r}^{r}-\frac{\lambda_{1} c_{0}}{q}\left\|u^{+}\right\|_{q}^{q} \\
& \text { for some } c_{25}>0\left(\text { since } \eta>\|\xi\|_{\infty}\right) \\
& \geq c_{25}\left\|u^{-}\right\|^{p}+\frac{1}{p}\left\|D u^{+}\right\|_{p}^{p}-\frac{1}{p}\|\xi\|_{\infty}\left\|u^{+}\right\|_{p}^{p}+c_{26}\left\|u^{+}\right\|_{p}^{r}-\frac{\lambda_{1} c_{0}}{q}\left\|u^{+}\right\|_{q}^{q} \\
& \text { for some } c_{26}>0(\text { recall that } p<r) \\
&= c_{25}\left\|u^{-}\right\|^{p}+\frac{1}{p}\left\|D u^{+}\right\|_{p}^{p}+\left[c_{26}\left\|u^{+}\right\|_{p}^{r-p}-\frac{1}{p}\|\xi\|_{\infty}\right]\left\|u^{+}\right\|_{p}^{p}-\frac{\lambda_{1} c_{0}}{q}\left\|u^{+}\right\|_{q}^{q} \\
&\Rightarrow d(\cdot) \text { is coercive (recall that } q<p<r) .
\end{aligned}
$$

Clearly, $d(\cdot)$ is also sequentially weakly lower semicontinuous. Thus, we can find $\widetilde{u} \in W^{1, p}(\Omega)$ such that

$$
d(\widetilde{u})=\inf \left[d(u): u \in W^{1, p}(\Omega)\right] .
$$

As before (see the proof of Proposition 3), since $q<p<r$, we have

$$
\begin{aligned}
& d(\widetilde{u})<0=d(0), \\
\Rightarrow \quad & \widetilde{u} \neq 0 .
\end{aligned}
$$

From (62), we have

$$
\begin{aligned}
& \langle A(\widetilde{u}), h\rangle+\int_{\Omega} \xi(z)|\widetilde{u}|^{p-2} \widetilde{u} h \mathrm{~d} z+\int_{\partial \Omega} \beta(z)|\widetilde{u}|^{p-2} \widetilde{u} h \mathrm{~d} \sigma-\int_{\Omega} \eta\left(\widetilde{u}^{-}\right)^{p-1} h \mathrm{~d} z \\
= & \int_{\Omega}\left[\lambda_{1} c_{0}\left(\widetilde{u}^{+}\right)^{q-1}-c_{24}\left(\widetilde{u}^{+}\right)^{r-1}\right] h \mathrm{~d} z \quad \text { for all } h \in W^{1, p}(\Omega) .
\end{aligned}
$$

In (63), we choose $h=-\widetilde{u} \in W^{1, p}(\Omega)$. Then,

$$
\begin{aligned}
& \gamma_{p}\left(\widetilde{u}^{-}\right)+\eta\left\|\widetilde{u}^{-}\right\|_{p}^{p}=0, \\
\Rightarrow & \widetilde{u} \geq 0, \widetilde{u} \neq 0 \quad\left(\text { recall that } \eta>\|\xi\|_{\infty}\right) .
\end{aligned}
$$

From (63) and (64), it follows that $\widetilde{u}$ is a positive solution of problem (61). Furthermore, the nonlinear regularity theory and the nonlinear Hopf boundary point theorem imply that $\widetilde{u} \in \operatorname{int} C_{+}$.

We claim that $\widetilde{u}$ is the unique solution of (61). To this end, suppose that $\widetilde{v}$ is another positive solution of (61). Again, we have $\widetilde{v} \in \operatorname{int} C_{+}$. Let $t>0$ be the biggest positive real such that

$$
t \widetilde{v} \leq \widetilde{u}
$$

(see [18], Proposition 4.1.22). Suppose that $t<1$. Let $\rho=\|\widetilde{u}\|_{\infty}$. We can find $\widetilde{\xi}_{\rho}>0$ such that

$$
x \mapsto \lambda_{1} c_{0} x^{q-1}-c_{24} x^{r-1}+\widetilde{\zeta}_{\rho} x^{p-1}
$$


is nondecreasing on $[0, \rho]$. We have

$$
\begin{aligned}
- & \Delta_{p}(t \widetilde{v})+\left[\xi(z)+\widetilde{\zeta}_{\rho}\right](t \widetilde{v})^{p-1} \\
& =t^{p-1}\left[-\Delta_{p} \widetilde{v}+\left(\widetilde{\zeta}(z)+\widetilde{\zeta}_{\rho}\right) \widetilde{v}^{p-1}\right] \\
& =t^{p-1}\left[\lambda_{1} c_{0} \widetilde{v}^{q-1}-c_{24} \widetilde{v}^{r-1}+\widetilde{\zeta}_{\rho} \widetilde{v}^{p-1}\right] \\
& \leq \lambda_{1} c_{0}(t \widetilde{v})^{q-1}-c_{24}(t \widetilde{v})^{r-1}+\widetilde{\zeta}_{\rho}(t \widetilde{v})^{p-1} \quad(\text { since } t<1, q<p<r) \\
& \leq \lambda_{1} c_{0} \widetilde{u}^{q-1}-c_{24} \widetilde{u}^{r-1}+\widetilde{\zeta}_{\rho} \widetilde{u}^{p-1} \quad(\text { see }(65)) \\
& =-\Delta_{p} \widetilde{u}+\left[\widetilde{\zeta}(z)+\widetilde{\zeta}_{\rho}\right] \widetilde{u}^{p-1} \quad \text { for a.a. } z \in \Omega .
\end{aligned}
$$

Let $\widetilde{m}=\min _{\bar{\Omega}} \widetilde{v}>0\left(\right.$ recall that $\left.\widetilde{v} \in \operatorname{int} C_{+}\right)$. We have

$$
c_{24} \widetilde{v}^{r-1}\left[t^{p-1}-t^{r-1}\right] \geq c_{24} \widetilde{m}^{r-1}\left[t^{p-1}-t^{r-1}\right]>0 .
$$

Thus, from (66) and Proposition 2.10 of Papageorgiou-Rădulescu-Repovš [25] (the strong comparison principle), we have

$$
\widetilde{u}-t \widetilde{v} \in \widehat{K}_{+}
$$

which contradicts the maximality of $t>0$ in (65). Hence, $t \geq 1$ and so

$$
\widetilde{v} \leq \widetilde{u}
$$

If in the above argument we reverse the roles of $\widetilde{v}$ and $\widetilde{u}$, we have

$$
\begin{aligned}
\widetilde{u} & \leq \widetilde{v}, \\
\Rightarrow \quad \widetilde{u} & =\widetilde{v}
\end{aligned}
$$

and this proves the uniqueness of the solution of (61).

Claim: $\widetilde{u} \leq u_{n}$ for all $n \in \mathbb{N}$.

For $n \in \mathbb{N}$, we consider the following Carathéodory function:

$$
\begin{aligned}
& i_{n}(z, x)= \begin{cases}\lambda_{1} c_{0}\left(x^{+}\right)^{q-1}-c_{24}\left(x^{+}\right)^{r-1}+\eta\left(x^{+}\right)^{p-1} & \text { if } x \leq u_{n}(z) \\
\lambda_{1} c_{0} u_{n}(z)^{q-1}-c_{24} u_{n}(z)^{r-1}+\eta u_{n}(z)^{p-1} & \text { if } u_{n}(z)<x\end{cases} \\
& \text { (recall that } \left.\eta>\|\xi\|_{\infty}\right)
\end{aligned}
$$

We set $I_{n}(z, x)=\int_{0}^{x} i_{n}(z, s) \mathrm{d} s$ and consider the $C^{1}$-functional

$$
\widehat{d}_{n}(u)=\frac{1}{p} \gamma_{p}(u)+\frac{\eta}{p}\|u\|_{p}^{p}-\int_{\Omega} I_{n}(z, u) \mathrm{d} z \quad \text { for all } u \in W^{1, p}(\Omega) .
$$

From (67) and since $\eta>\|\xi\|_{\infty}$, we see that $\widehat{d}_{n}(\cdot)$ is coercive. In addition, it is sequentially weakly lower semicontinuous. Thus, we can find $\bar{u}_{n} \in W^{1, p}(\Omega)$ such that

$$
\begin{aligned}
& \left.\widehat{d}_{n}\left(\bar{u}_{n}\right)=\inf \left[\widehat{d}_{n}(u): u \in W^{1, p}(\Omega)\right]<0=\widehat{d}_{n}(0) \quad \text { (since } q<p<r\right) \\
\Rightarrow & \bar{u}_{n} \neq 0 .
\end{aligned}
$$

We have

$$
\widehat{d}_{n}\left(\bar{u}_{n}\right)=0,
$$




$$
\begin{gathered}
\Rightarrow \quad\left\langle A\left(\bar{u}_{n}\right), h\right\rangle+\int_{\Omega}[\xi(z)+\eta]\left|\bar{u}_{n}\right|^{p-2} \bar{u}_{n} h \mathrm{~d} z+\int_{\partial \Omega} \beta(z)\left|\bar{u}_{n}\right|^{p-2} \bar{u}_{n} h \mathrm{~d} \sigma \\
=\int_{\Omega} i\left(z, \bar{u}_{n}\right) h \mathrm{~d} z \quad \text { for all } h \in W^{1, p}(\Omega) .
\end{gathered}
$$

In (68), we choose $h=-\bar{u}_{n}^{-} \in W^{1, p}(\Omega)$ and then $h=\left(\bar{u}_{n}-u_{n}\right)^{+} \in W^{1, p}(\Omega)$ and we obtain

$$
\begin{aligned}
& \bar{u}_{n} \in\left[0, u_{n}\right], \bar{u}_{n} \neq 0, \\
\Rightarrow & \left.\bar{u}_{n} \text { is a positive solution of (61) (see }(67),(69)\right), \\
\Rightarrow & \bar{u}_{n}=\widetilde{u} \in \operatorname{int} C_{+} .
\end{aligned}
$$

Therefore, $\tilde{u} \leq u_{n}$ for all $n \in \mathbb{N}$, and this proves the Claim.

Passing to the limit as $n \rightarrow \infty$ in (57) and using (59) and the Claim, we obtain

$$
\left\langle A\left(u_{*}\right), h\right\rangle+\int_{\Omega} \xi(z) u_{*}^{p-1} h \mathrm{~d} z+\int_{\partial \Omega} \beta(z) u_{*}^{p-1} h \mathrm{~d} \sigma=\int_{\Omega}\left[\lambda^{*} g\left(z, u_{*}\right)+f\left(z, u_{*}\right)\right] h \mathrm{~d} z
$$

for all $h \in W^{1, p}(\Omega)$,

$\widetilde{u} \leq u_{*}$.

Then, (72) and (73) imply

$$
\begin{aligned}
& u_{*} \in \mathscr{S}_{\lambda^{*}} \subseteq \operatorname{int} C_{+} \\
\Rightarrow \quad & \lambda^{*} \in \mathscr{L} .
\end{aligned}
$$

From Propositions 3 and 7, we have

$$
\mathscr{L}=\left(0, \lambda^{*}\right]
$$

\subsection{Multiplicity of Positive Solutions}

Here, we show that, if $\lambda \in\left(0, \lambda^{*}\right)$, then problem (1) admits two positive solutions. In fact, the pair is also ordered.

Proposition 8. If hypotheses $\mathrm{H}(\xi), \mathrm{H}(\beta), \mathrm{H}(g), \mathrm{H}(f), \mathrm{H}_{0}$ hold and $\lambda \in\left(0, \lambda^{*}\right)$, then problem (1) has at least two positive solutions

$$
u_{0}, \widehat{u} \in \operatorname{int} C_{+}, \quad u_{0} \leq \widehat{u}, u_{0} \neq \widehat{u}
$$

Proof. Let $\mu \in\left(\lambda, \lambda^{*}\right)$. Then, $\mu \in \mathscr{L}$ (see (76)) and we can find $u_{\mu} \in \mathscr{S}_{\mu} \subseteq$ int $C_{+}$. On account of Proposition 4, we can find $u_{0} \in \mathscr{S}_{\lambda} \subseteq$ int $C_{+}$such that

$$
u_{0} \in \operatorname{int}_{C^{1}(\bar{\Omega})}\left[0, u_{\mu}\right]
$$

Moreover, $u_{0}$ is the minimizer of the functional $\widehat{\psi}_{\lambda}(\cdot)$ from the proof of Proposition 3.

We have that (see Papageorgiou-Rădulescu [20], Proposition 2.12)

$$
\begin{aligned}
& \left.\varphi_{\lambda}\right|_{\left[0, u_{\mu}\right]}=\left.\widehat{\psi}_{\lambda}\right|_{\left[0, u_{\mu}\right]} \quad \text { (see the proof of Proposition 7), } \\
\Rightarrow \quad & u_{0} \text { is a local } C^{1}(\bar{\Omega}) \text {-minimizer of } \varphi_{\lambda}(\text { see }(77)) \\
\Rightarrow \quad & u_{0} \text { is a local } W^{1, p}(\Omega) \text {-minimizer of } \varphi_{\lambda} .
\end{aligned}
$$


Consider the following Carathéodory function

$$
\begin{aligned}
& k_{\lambda}(z, x)= \begin{cases}\lambda g\left(z, u_{0}(z)\right)+f\left(z, u_{0}(z)\right)+\eta u_{0}(z)^{p-1} & \text { if } x \leq u_{0}(z) \\
\lambda g(z, x)+f(z, x)+\eta x^{p-1} & \text { if } u_{0}(z)<x\end{cases} \\
& \text { (as before } \left.\eta>\|\xi\|_{\infty}\right) .
\end{aligned}
$$

We set $K_{\lambda}(z, x)=\int_{0}^{x} k_{\lambda}(z, s)$ ds and introduce the $C^{1}$-functional $j_{\lambda}: W^{1, p}(\Omega) \rightarrow \mathbb{R}$ defined by

$$
j_{\lambda}(u)=\frac{1}{p} \gamma_{p}(u)+\frac{\eta}{p}\|u\|_{p}^{p}-\int_{\Omega} K_{\lambda}(z, u) \mathrm{d} z \quad \text { for all } u \in W^{1, p}(\Omega) .
$$

Using (78), we can easily show that

$$
\left.K_{j_{\lambda}} \subseteq\left[u_{0}\right) \cap \operatorname{int} C_{+} \quad \text { and } \quad u_{0} \in K_{j_{\lambda}} \text { (since } u_{0} \in \mathscr{S}_{\lambda}\right) .
$$

On account of (79), we may assume that

$$
K_{j_{\lambda}} \cap\left[u_{0}, u_{\mu}\right]=\left\{u_{0}\right\} .
$$

Otherwise, we already have a second positive solution of (1), and this new solution is bigger than $u_{0}$. Thus, we are done.

Reasoning as in the Claim in the proof of Proposition 3, we show that

$$
j_{\lambda} \text { satisfies the C-condition. }
$$

We truncate $k_{\lambda}(z, \cdot)$ at $u_{\mu}(z)$ and produce a new Carathéodory function $\widehat{k}_{\lambda}(z, x)$. We set $\widehat{K}_{\lambda}(z, x)=$ $\int_{0}^{x} \widehat{k}_{\lambda}(z, s) \mathrm{d} s$ and introduce the $C^{1}$-functional $\hat{j}_{\lambda}: W^{1, p}(\Omega) \rightarrow \mathbb{R}$ defined by

$$
\widehat{j}_{\lambda}(u)=\frac{1}{p} \gamma_{p}(u)+\frac{\eta}{p}\|u\|_{p}^{p}-\int_{\Omega} \widehat{K}_{\lambda}(z, u) \mathrm{d} z \quad \text { for all } u \in W^{1, p}(\Omega) .
$$

We can easily show that

$$
K_{\widehat{j_{\lambda}}}=K_{j_{\lambda}} \cap\left[u_{0}, u_{\mu}\right] .
$$

The functional $\widehat{j_{\lambda}}(\cdot)$ is coercive and sequentially weakly lower semicontinuous. Hence, we can find $\widehat{u}_{0} \in W^{1, p}(\Omega)$ such that

$$
\begin{aligned}
& \widehat{j}_{\lambda}\left(\widehat{u}_{0}\right)=\inf \left[\widehat{j}_{\lambda}(u): u \in W^{1, p}(\Omega)\right], \\
\Rightarrow & \widehat{u}_{0} \in K_{\widehat{j}_{\lambda}^{\prime}} \\
\Rightarrow & \widehat{u}_{0}=u_{0}(\operatorname{see}(80),(82)) .
\end{aligned}
$$

Evidently, we have

$$
\left.j_{\lambda}\right|_{\left[0, u_{\mu}\right]}=\left.\widehat{j}_{\lambda}\right|_{\left[0, u_{\mu}\right]} .
$$

From (77), (84) and [20], it follows that

$$
\Rightarrow \begin{aligned}
& u_{0} \text { is a local } C^{1}(\bar{\Omega}) \text {-minimizer of } j_{\lambda}, \\
& \quad u_{0} \text { is a local } W^{1, p}(\Omega) \text {-minimizer of } j_{\lambda} .
\end{aligned}
$$

From (79), it is clear that we may assume that

$$
K_{j_{\lambda}} \text { is finite. }
$$


Otherwise, on account of (78), we already have a sequence of critical points of $j_{\lambda}(\cdot)$ which are positive solutions of (1), all bigger that $u_{0}$ and so we are done.

Now, (85), (86), and Theorem 5.7.6 of Papageorgiou-Rădulescu-Repovš [18] imply that we can find $\rho \in(0,1)$ small such that

$$
j_{\lambda}\left(u_{0}\right)<\inf \left[j_{\lambda}(u):\left\|u-u_{0}\right\|=\rho\right]=m_{\lambda} .
$$

Moreover, hypotheses $\mathrm{H}(g)$ (ii) and $\mathrm{H}(f)$ (ii) imply that, for $u \in \operatorname{int} C_{+}$, we have

$$
j_{\lambda}(t u) \rightarrow-\infty \text { as } t \rightarrow+\infty .
$$

Then, (81), (87), and (88) permit the use of the mountain pass theorem. Thus, we can find $\widehat{u} \in W^{1, p}(\Omega)$ such that

$$
\widehat{u} \in K_{j_{\lambda}} \subseteq\left[u_{0}\right) \cap \operatorname{int} C_{+} \quad(\text { see (79)) }
$$

and

$$
j_{\lambda}\left(u_{0}\right)<m_{\lambda} \leq j_{\lambda}(\widehat{u})(\text { see }(87)) .
$$

From these facts and (78), we conclude that

$$
\widehat{u} \in \mathscr{S}_{\lambda} \subseteq \operatorname{int} C_{+}, \quad u_{0} \leq \widehat{u}, u_{0} \neq \widehat{u} .
$$

\subsection{Minimal Positive Solutions}

In this subsection, we show that, for every $\lambda \in \mathscr{L}$, problem (1) has a smallest positive solution $u_{\lambda}^{*}$ and examine the properties of the map $\lambda \mapsto u_{\lambda}^{*}$.

Proposition 9. If hypotheses $\mathrm{H}(\xi), \mathrm{H}(\beta), \mathrm{H}(g), \mathrm{H}(f), \mathrm{H}_{0}$ hold and $\lambda \in \mathscr{L}=\left(0, \lambda^{*}\right]$, then problem(1) has a smallest positive solution $u_{\lambda}^{*} \in \mathscr{S}_{\lambda} \subseteq \operatorname{int} C_{+}$(that is, $u_{\lambda}^{*} \leq u$ for all $u \in \mathscr{S}_{\lambda}$ ).

Proof. From Papageorgiou-Rădulescu-Repovš [27] (proof of Proposition 7), we know that $\mathscr{S}_{\lambda}$ is downward directed (that is, if $u_{1}, u_{2} \in \mathscr{S}_{\lambda}$, then we can find $u \in \mathscr{S}_{\lambda}$ such that $u \leq u_{1}, u \leq u_{2}$ ). Invoking Lemma 3.10, p. 178, of Hu-Papageorgiou [28], we can find a decreasing sequence $\left\{u_{n}\right\}_{n \geq 1} \subseteq$ $\mathscr{S}_{\lambda}$ such that

$$
\inf \mathscr{S}_{\lambda}=\inf _{n \geq 1} u_{n}
$$

We have

$$
\begin{aligned}
& \left\langle A\left(u_{n}\right), h\right\rangle+\int_{\Omega} \xi(z) u_{n}^{p-1} h \mathrm{~d} z+\int_{\partial \Omega} \beta(z) u_{n}^{p-1} h \mathrm{~d} \sigma=\int_{\Omega}\left[\lambda g\left(z, u_{n}\right)+f\left(z, u_{n}\right)\right] h \mathrm{~d} z \\
& \text { for all } h \in W^{1, p}(\Omega), \text { all } n \in \mathbb{N} .
\end{aligned}
$$

Since $0 \leq u_{n} \leq u_{1}$ for all $n \in \mathbb{N}$, choosing $h=u_{n} \in W^{1, p}(\Omega)$ in (89), we infer that

$$
\left\{u_{n}\right\}_{n \geq 1} \subseteq W^{1, p}(\Omega) \text { is bounded. }
$$

Thus, we may assume that

$$
u_{n} \stackrel{w}{\rightarrow} u_{\lambda}^{*} \text { in } W^{1, p}(\Omega) \quad \text { and } \quad u_{n} \rightarrow u_{\lambda}^{*} \text { in } L^{r}(\Omega) \text { and in } L^{p}(\partial \Omega) .
$$


In (89), we choose $h=u_{n}-u_{\lambda}^{*} \in W^{1, p}(\Omega)$, pass to the limit as $n \rightarrow \infty$ and use (89). We obtain

$$
\begin{aligned}
& \lim _{n \rightarrow \infty}\left\langle A\left(u_{n}\right), u_{n}-u_{\lambda}^{*}\right\rangle=0, \\
\Rightarrow & u_{n} \rightarrow u_{\lambda}^{*} \quad \text { in } W^{1, p}(\Omega) .
\end{aligned}
$$

Thus, if in (89) we pass to the limit as $n \rightarrow \infty$ and use (92), we obtain

$$
\begin{aligned}
& \left\langle A\left(u_{\lambda}^{*}\right), h\right\rangle+\int_{\Omega} \xi(z)\left(u_{\lambda}^{*}\right)^{p-1} h \mathrm{~d} z+\int_{\partial \Omega} \beta(z)\left(u_{\lambda}^{*}\right)^{p-1} h \mathrm{~d} \sigma \\
= & \int_{\Omega}\left[\lambda g\left(z, u_{\lambda}^{*}\right)+f\left(z, u_{\lambda}^{*}\right)\right] h \mathrm{~d} z \quad \text { for all } h \in W^{1, p}(\Omega) .
\end{aligned}
$$

Moreover, from the proof of Proposition 7 (see the Claim), we have

$$
\widetilde{u} \leq u_{\lambda}^{*}
$$

(in this case, in problem (61) $\lambda_{1}$ is replaced by $\lambda$ ). Then, (93) and (94) imply that $u_{\lambda}^{*} \in \mathscr{S}_{\lambda} \subseteq \operatorname{int} C_{+}$and $u_{\lambda}^{*}=\inf \mathscr{S}_{\lambda}$.

We examine the properties of the map $\lambda \mapsto u_{\lambda}^{*}$.

Proposition 10. If hypotheses $\mathrm{H}(\xi), \mathrm{H}(\beta), \mathrm{H}(g), \mathrm{H}(f), \mathrm{H}_{0}$ hold, then the minimal solution map $\lambda \mapsto u_{\lambda}^{*}$ from $\mathscr{L}=\left(0, \lambda^{*}\right]$ into $C^{1}(\bar{\Omega})$ has the following properties:

(a) it is strictly increasing in the sense that

$$
0<\mu<\lambda<\lambda^{*} \quad \Rightarrow \quad u_{\lambda}^{*}-u_{\mu}^{*} \in \widehat{K}_{+}
$$

(b) it is left continuous.

\section{Proof.}

(a) According to Proposition 4, we can find $u_{\mu} \in \mathscr{S}_{\mu} \subseteq \operatorname{int} C_{+}$such that

$$
\begin{aligned}
& u_{\lambda}^{*}-u_{\mu} \in \widehat{K}_{+} \\
\Rightarrow \quad & u_{\lambda}^{*}-u_{\mu}^{*} \in \widehat{K}_{+} \quad\left(\text { since } u_{\mu}^{*} \leq u_{\mu}\right) .
\end{aligned}
$$

(b) Let $\left\{\lambda_{n}\right\}_{n \geq 1} \subseteq \mathscr{L}=\left(0, \lambda^{*}\right]$ and assume that $\lambda_{n} \rightarrow \lambda^{-}$. Then,

$$
\left\{u_{\lambda_{n}}^{*}\right\}_{n \geq 1} \subseteq W^{1, p}(\Omega) \text { is bounded. }
$$

Using (95) and since $u_{\lambda_{n}}^{*} \in \mathscr{S}_{\lambda_{n}}$ for all $n \in \mathbb{N}$, as in the last part of the proof of Proposition 2 (see the part of the proof after (29)), we obtain

$$
\begin{aligned}
& u_{\lambda_{n}}^{*} \rightarrow \widetilde{u}_{\lambda}^{*} \operatorname{in} W^{1, p}(\Omega), \\
\Rightarrow & \widetilde{u}_{\lambda}^{*} \in \mathscr{S}_{\lambda} \subseteq \operatorname{int} C_{+} .
\end{aligned}
$$

We will show that $\widetilde{u}_{\lambda}^{*}=u_{\lambda}^{*}$. If this inequality is not true, we can find $z_{0} \in \bar{\Omega}$ such that

$$
u_{\lambda}^{*}\left(z_{0}\right)<\widetilde{u}_{\lambda}^{*}\left(z_{0}\right) .
$$


From (95) and Proposition 2.10 of Papageorgiou-Rădulescu [20], we have

$$
\left\|u_{\lambda_{n}}^{*}\right\| \leq c_{25} \quad \text { for some } c_{25}>0 \text {, all } n \in \mathbb{N} \text {. }
$$

Then, Theorem 2 of Lieberman [21] implies that there exist $\alpha \in(0,1)$ and $c_{26}>0$ such that

$$
u_{\lambda_{n}}^{*} \in C^{1, \alpha}(\bar{\Omega}) \quad \text { and } \quad\left\|u_{\lambda_{n}}^{*}\right\|_{C^{1, \alpha}(\bar{\Omega})} \leq c_{26} \text { for all } n \in \mathbb{N} \text {. }
$$

The compact embedding of $C^{1, \alpha}(\bar{\Omega})$ into $C^{1}(\bar{\Omega})$ and (96) implies that

$$
\begin{aligned}
& u_{\lambda_{n}}^{*} \rightarrow \widetilde{u}_{\lambda}^{*} \text { in } C^{1}(\bar{\Omega}), \\
\Rightarrow \quad & u_{\lambda}^{*}\left(z_{0}\right)<u_{\lambda_{n}}^{*}\left(z_{0}\right) \text { for all } n \geq n_{0}(\text { see }(97))
\end{aligned}
$$

However, this contradicts (a). Thus, $\widetilde{u}_{\lambda}^{*}=u_{\lambda}^{*}$ and we conclude that $\lambda \mapsto u_{\lambda}^{*}$ is left continuous.

\section{Main Theorem-Conclusions}

Thus, summarizing our findings in this paper, we can state the following theorem.

Theorem 1. If hypotheses $\mathrm{H}(\xi), \mathrm{H}(\beta), \mathrm{H}(g), \mathrm{H}(f), \mathrm{H}_{0}$ hold, then there exists a critical parameter value $\lambda^{*}$ such that

(a) for all $\lambda \in\left(0, \lambda^{*}\right)$, problem (1) has at least two positive solutions

$$
u_{0}, \widehat{u} \in \operatorname{int} C_{+}, \quad u_{0} \leq \widehat{u}, \quad u_{0} \neq \widehat{u}
$$

(b) for $\lambda=\lambda^{*}$, problem (1) has at least one positive solution $u_{*} \in \operatorname{int} C_{+}$;

(c) for all $\lambda>\lambda^{*}$, there are no positive solutions for problem (1);

(d) for all $\lambda \in\left(0, \lambda^{*}\right]$, problem (1) has a smallest positive solution $u_{\lambda}^{*} \in \operatorname{int} C_{+}$and the map $\lambda \mapsto u_{\lambda}^{*}$ from $\mathscr{L}=\left(0, \lambda^{*}\right]$ and $C^{1}(\bar{\Omega})$ is $\widehat{K}_{+}$-striclty increasing and left continuous.

In this paper, we have investigated a very general version of the "convex-concave problem". As it was illustrated in earlier works (see, for example, $[2,4-6,8,10]$ ), such problems exhibit interesting mathematical features. In the past, most of the works deal with Dirichlet problems with no potential term. This way, they have a coercive differential operator and this fact facilitates the analysis of the problem. Here, we have a Robin boundary condition and an indefinite potential term. Moreover, the reaction (source term) is much more general and not necessarily of polynomial type. In most of the previous works, the reaction is of the form

$$
x \mapsto \lambda x^{q-1}+x^{r-1} \quad \text { for } x \geq 0
$$

with $1<q<p<r<p^{*}$. Our reaction term is much more general. It will be interesting to extend our work here to double-phase equation with the concave contribution coming from the boundary condition. A first step in this direction was made by the authors for $(p, 2)$-equations with a positive potential term (see [10]). We point out that double-phase problems $((p, q)$-equations) arise in many mathematical models of physical processes.

Author Contributions: All authors contributed equally to this paper. All authors have read and agreed to the published version of the manuscript.

Funding: This research received no external funding.

Acknowledgments: The authors wish to thank the anonymous reviewers for their remarks and constructive criticism which helped them to improve the presentation. 
Conflicts of Interest: The authors declare no conflict of interest.

\section{References}

1. Gasiński, L.; Papageorgiou, N.S. Nonlinear Analysis; Chapman\&Hall/CRC: Boca Raton, FL, USA, 2006.

2. Papageorgiou, N.S.; Rădulescu, V.D.; Repovš, D. Robin problems with and indefinite linear part and competition phenomena. Commun. Pure Appl. Anal. 2017, 16, 1293-1314. [CrossRef]

3. Marano, S.A.; Marino, G.; Papageorgiou, N.S. On a Dirichlet problem with $(p, q)$-Laplacian and parametric concave-convex nonlinearity. J. Math. Anal. Appl. 2019, 475, 1093-1107. [CrossRef]

4. Ambrosetti, A.; Brezis, H.; Cerami, G. Combined effects of concave and convex nonlinearities in some elliptic problems. J. Funct. Anal. 1994, 122, 519-543. [CrossRef]

5. García Azorero, J.P.; Peral Alonso, I.; Manfredi, J.J. Sobolev versus Hölder local minimizers and global multiplicity for some quasi-linear elliptic equations. Commun. Contemp. Math. 2000, 2, 385-404. [CrossRef]

6. Guo, Z.; Zhang, Z. $W^{1, p}$ versus $C^{1}$ local minimizers and multiplicity results for quasilinear elliptic equations. J. Math. Anal. Appl. 2003, 286, 32-50. [CrossRef]

7. $\mathrm{Hu}, \mathrm{S}$; Papageorgiou, N.S. Multiplicity of solutions for parametric $p$-Laplacian equations with nonlinearity concave near the origin. Tohoku Math. J. 2010, 62, 137-162. [CrossRef]

8. Marano, S.A.; Papageorgiou, N.S. Positive solutions to a Dirichlet problem with $p$-Laplacian and concave-convex nonlinearity depending on a parameter. Commun. Pure Appl. Anal. 2013, 12, 815-829. [CrossRef]

9. Papageorgiou, N.S.; Scapellato, A. Nonlinear Robin problems with general potential and crossing reaction. Rend. Lincei Mat. Appl. 2019, 30, 1-29. [CrossRef]

10. Papageorgiou, N.S.; Scapellato, A. Positive solutions for $(p, 2)$-equations with superlinear reaction and a concave boundary term. Electron. J. Qual. Theory Differ. Equ. 2020, 2020, 1-19. [CrossRef]

11. DiBenedetto, E.; Gianazza, U.; Vespri, V. Remarks on Local Boundedness and Local Holder Continuity of Local Weak Solutions to Anisotropic p-Laplacian Type Equations. J. Elliptic Parabol. Equ. 2016, 2, 157-169. [CrossRef]

12. Drábek, P.; Hernández, J. Quasilinear eigenvalue problems with singular weights for the $p$-Laplacian. Ann. Mat. Pura Appl. 2019, 198, 1069-1086. [CrossRef]

13. Drábek, P.; Ho, K.; Sarkar, A. On the eigenvalue problem involving the weighted $p$-Laplacian in radially symmetric domains. J. Math. Anal. Appl. 2018, 468, 716-756. [CrossRef]

14. Drábek, P.; Ho, K.; Sarkar, A. The Fredholm alternative for the $p$-Laplacian in exterior domains. Nonlinear Anal.-Theory Methods Appl. 2018, 174, 17-53. [CrossRef]

15. Drábek, P.; Pohozaev, S.I. Positive solutions for the $p$-Laplacian: Application of the fibrering method. R. Soc. Edinb.-Proc. A 1997, 127, 703-726. [CrossRef]

16. Jebelean, P.; Mawhin, J.; Şerban, C. Morse theory and multiple periodic solutions of some quasilinear difference systems with periodic nonlinearities. Georgian Math. J. 2017, 24, 103-112. [CrossRef]

17. Manásevich, R.; Mawhin, J. The spectrum of $p$-Laplacian systems with various boundary conditions and applications. Adv. Differ. Equ. 2000, 5, 1289-1318.

18. Papageorgiou, N.S.; Rădulescu, V.D.; Repovš, D. Nonlinear Analysis-Theory and Methods; Springer: New York City, NY, USA, 2019.

19. Ambrosetti, A.; Rabinowitz, P. Dual variational methods in critical point theory and applications. J. Funct. Anal. 1973, 14, 349-381. [CrossRef]

20. Papageorgiou, N.S.; Rădulescu, V.D. Nonlinear nonhomogeneous Robin problems with superlinear reaction term. Adv. Nonlinear Stud. 2016, 16, 737-764. [CrossRef]

21. Lieberman, G. Boundary regularity for solutions of degenerate elliptic equations. Nonlinear Anal. 1988, 12, 1203-1219. [CrossRef]

22. Pucci, P.; Serrin, J. The Maximum Principle; Birkhäuser: Basel, Switzerland, 2007.

23. Papageorgiou, N.S.; Winkert, P. Applied Nonlinear Functional Analysis; W. De Gruyter: Berlin, Germany, 2018.

24. Papageorgiou, N.S.; Rădulescu, V.D. Multiple solutions with precise sign information for nonlinear parametric Robin problems. J. Differ. Equ. 2014, 256, 2449-2479. [CrossRef]

25. Papageorgiou, N.S.; Rădulescu, V.D.; Repovš, D. Positive solutions for nonlinear nonhomogeneous parametric Robin problems. Forum Math. 2018, 30, 553-580. [CrossRef] 
26. Godoy, T.; Gossez, J.-P.; Paczka, S. On the antimaximum principle for the $p$-Laplacian with indefinite weight. Nonlin. Anal. 2002, 51, 449-467. [CrossRef]

27. Papageorgiou, N.S.; Rădulescu, V.D.; Repovš, D. Positive solutions for perturbations of the Robin eigenvalue problem plus and indefinite potential. Discret. Contin. Dyn. Syst.-A 2017, 37, 2589-2618. [CrossRef]

28. Hu, S.; Papageorgiou, N.S. Handbook of Multivalued Analysis: Volume I: Theory; Kluwer Academic Publishers: Dordrecht, The Netherlands, 1997.

(C) 2020 by the authors. Licensee MDPI, Basel, Switzerland. This article is an open access article distributed under the terms and conditions of the Creative Commons Attribution (CC BY) license (http://creativecommons.org/licenses/by/4.0/). 